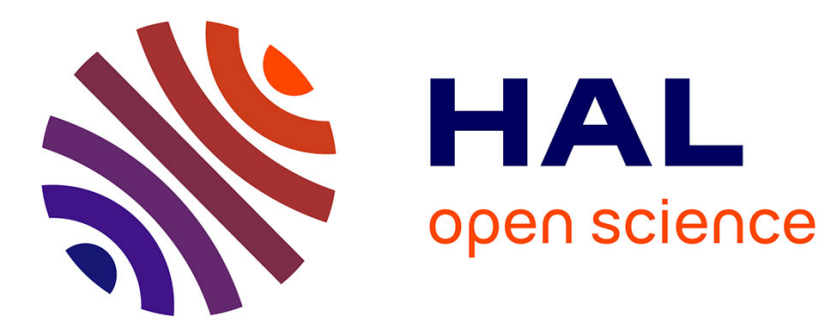

\title{
Couche épitaxique mince sur un substrat semi-infini: rôle du désaccord paramétrique et de l'épaisseur sur les distortions élastiques
}

R. Bonnet, Jean-Louis Verger-Gaugry

\section{- To cite this version:}

R. Bonnet, Jean-Louis Verger-Gaugry. Couche épitaxique mince sur un substrat semi-infini: rôle du désaccord paramétrique et de l'épaisseur sur les distortions élastiques. Philosophical Magazine a, 1992, 66 (5), pp.849-871. hal-03135968

\section{HAL Id: hal-03135968 \\ https://hal.science/hal-03135968}

Submitted on 13 Feb 2021

HAL is a multi-disciplinary open access archive for the deposit and dissemination of scientific research documents, whether they are published or not. The documents may come from teaching and research institutions in France or abroad, or from public or private research centers.
L'archive ouverte pluridisciplinaire HAL, est destinée au dépôt et à la diffusion de documents scientifiques de niveau recherche, publiés ou non, émanant des établissements d'enseignement et de recherche français ou étrangers, des laboratoires publics ou privés. 
Couche épitaxique mince sur un substrat semi-infini : rôle du désaccord paramétrique et de l'épaisseur sur les distortions élastiques

\author{
R. Bonnet and J.-L. Verger-Gaugry
}

LTPCM/ENSEEG/INPG (CNRS UA 29), BP 75, Domaine Universitaire, 38402 Saint Martin d'Hères, France 
The elastic deformation field associated with a thin crystal epitaxially deposited on a semi-infinite single-crystal substrate of different nature is determined by a method previously proposed by one of the authors for the case of two semi-infinite media, with isotropic elasticity. Two assumptions are envisaged for the calculation in the frame of the planar deformation, the stress field being periodic along a direction parallel to the interface for both cases. First, it is assumed that misfit dislocations (or intrinsic dislocations) are present and regularly spaced. Second, it is supposed that translation dislocations (or extrinsic dislocations) are introduced by plastic deformation. The displacement fields of the two kinds of dislocation, expressed relative to a Cartesian frame linked to the interface, differ only by a linear function of the coordinates. Expressions for them are explicitly formulated from the analytical inversion of a system of nine linear complex equations with nine unknowns. Detailed expressions for the stresses and volumic dilatations are presented.

For the case of a single family of intrinsic dislocations, the elastic energy stored in the material is calculated explicitly against $h$. Results are extended to a square network of edge intrinsic dislocations with numerical applications to the systems $\mathrm{GaSb} / \mathrm{GaAs}$ and $\mathrm{GaAs} / \mathrm{Si}$. 
Le champ des déformations élastiques associé à un cristal mince en épitaxie sur un cristal de nature différente, monocristallin et semi-infini, est déterminé en utilisant une méthode antérieurement proposée par l'un des auteurs dans le cas de deux milieux semi-infinis à élasticité isotrope. Deux hypothèses de calcul sont envisagées dans le cadre de la déformation plane, le champ des contraintes étant dans les deux cas périodique dans une direction parallèle à l'interface. L'une admet la présence de dislocations de désaccord paramétrique (ou dislocations intrinsèques) régulièrement réparties; l'autre suppose la présence de dislocations de translation (ou dislocations extrinsèques) introduites par déformation plastique. Les champs des déplacements de ces deux types de dislocations, exprimés dans un repère Cartésien lié à l'interface, ne diffèrent que d'une fonction linéaire des coordonnées. Leurs expressions sont rendues explicites en inversant analytiquement un système de neuf équations linéaires complexes à neuf inconnues. Les expressions détaillées des contraintes et dilatations volumiques sont présentées.

Dans le cas d'une famille de dislocations intrinsèques, l'énergie élastique stockée dans le matériau est calculée explicitement en fonction de $h$. Les résultats sont étendus à un réseau carré de dislocations intrinsèques coin, avec des applications numériques aux systèmes $\mathrm{GaSb} / \mathrm{GaAs}$ et $\mathrm{GaAs} / \mathrm{Si}$. 


\section{§1. INTRODUCTION}

Depuis environ une décennie, les études expérimentales des couches épitaxiques sur substrat monocristallin se sont développées considérablement, depuis les premiers instants de la nucléation jusqu'à l'obtention de l'épaisscur désiréc, celle-ci dépassant en général $10 \mathrm{~nm}$ dans les applications sur substrat de silicium (par exemple Cherns, Smith, Krakow et Batson 1982), Gibson, Tung, Phillips et Hull (1985), Catana (1990), Catana et al. (1990), Feuillet (1990) et Rocher, Da Silva et Raisin 1990b)). Lorsque ces couches ont des épaisseurs suffisantes, les interfaces sont souvent tapissées de dislocations disposées plus ou moins régulièrement suivant l'orientation cristallographique de la face du substrat et les traitements de recuit subis par l'échantillon. La densité et le caractère de ces dislocations dépendent des écarts paramétriques et angulaires entre les cristaux le long de l'interface, mais aussi de la rapidité avec laquelic a èté refroidi l'échantillon. Pour des cristaux cubiques en épitaxic, l'écart paramétrique ('lattice mismatch' ou 'Vernier') peut atteindre 15\% en étant accommodé par des dislocations dont les cocurs sont bien observables en microscopic électronique à haute résolution voir par exemple les dislocations de Lomer visibles aux interfaces $\mathrm{CdTe}(001) / \mathrm{GaAs}(001)$ (Feuillet 1990). Les champs des déplacements élastiques régnant au voisinage de ces interfaces sont étudiés en microscopic électronique à transmission (par exemple Cherns et al. (1982), Bulle-Licuwma, van Ommen et Hornstra (1988), Rocher et al. (1990b) et Tietz, Summerfelt et Carter (1990)) et par diffraction de rayons X (Bourret, Fuoss, Rocher et Raisin 1991). Il est donc nécessaire de disposer de modèles aussi réalistes que possible qui puissent décrire ces champs de déplacements en fonction de l'épaisseur $h$ de la couche déposéc, surtout lorsque la distance moyenne entre les dislocations d'interface et l'épaisseur $h$ sont du même ordre de grandeur (fig. 1).

Les premiers, Frank et van der Merwe (1949) se sont attaqués au problème de l'épitaxic en utilisant les propriétés élastiques des cristaux et les désaccords paramétrique des réseaux cristallins. Plus récemment, dans trois articles successils, van der Merwe (1982a, b, c) a amélioré son modèle en tenant compte de l'effet de l'épaisseur sur l'énergic élastique stockéc dans le matériau, et présente des applications aux accolements de faces (111) de cristaux c.f.c. sur des faces (110) de cristaux c.c. Dans le cas

Fig. 1

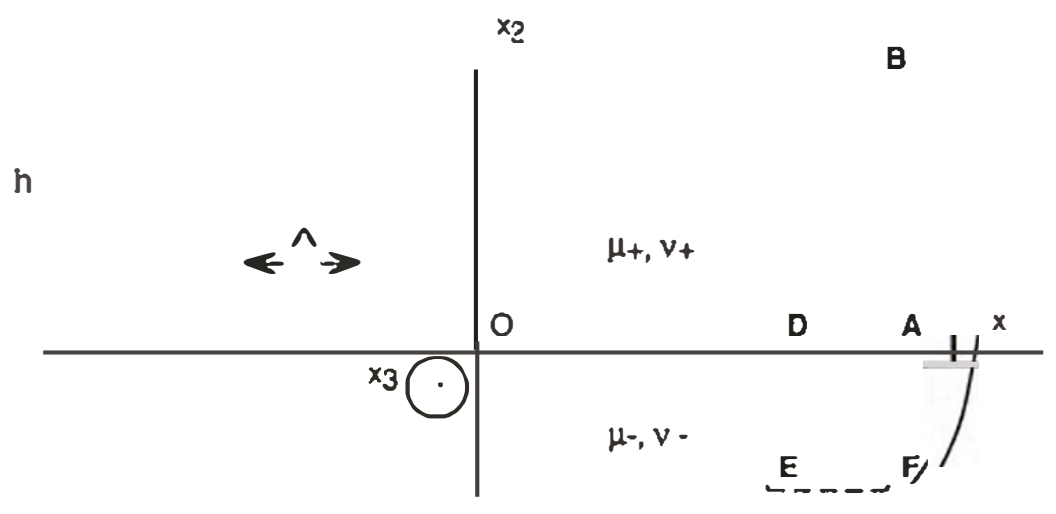

Schéma d'une couche monocristalline, d’épaisseur $h$, en épitaxie sur un substrat monocristallin. La période des dislocations interfaciales est $A$. Les modules de cisaillement et de Puissun sont quelconques. Le volume parallélépipédique ABCDEF entourant une singularité de déformation (coeur d'une dislocation) est en équilibre. 
particulier d'une épitaxie liée à des dislocations de désaccord paramétrique ('dislocations de Vernier' ou 'dislocations intrinsèques') $\propto$ modèle a œependant l'inconvénient d'utiliser un paramètre mal défini (un module de cisaillement propre à l'interface) et de donner une expression de l'énergie élastique emmagasinée qui ne dépend pas de rayons de coupure, ni d'un éventuel caractère mixte des dislocations. L'emploi d'un module de cisaillement interfacial est d'autant plus génant que ce modèle suppose que les cristaux conservent leurs structures atomiques jusqu'à l'interface elle-méme (van der Merwe 1982a), c’est-à-dire leurs propriétés élastiques respectives.

Dans un article récent, Willis, Jain et Bullough (1990) abordent ce problème, dans le cadre de la déformation plane, en admettant que les dislocations interfaciales sont des dislocations extrinsèques provenant de la déformation plastique de la couche épitaxique. Ils prennent en compte, pour la première fois, le problème de l'interaction du champ élastique de ces dislocations avec la surface libre, lorsque le joint interphase sépare deux milieux élastiquement isotropes, mais différents; leurs résultats ne sont fonction que des coefficients de Lamé des deux matériaux et des grandeurs géométriques du système. Ils admettent que linterface est abrupte en $\propto$ qui concerne les propriétés élastiques. Ils parviennent à formuler les contraintes et l'énergie élastique emmagasinće dans le matériau, mais sous la forme de séries dont les coefficients ne sont pas déterminés analytiquement dans le cas général. Dans le cas particulier où les deux milieux ont les mêmes propriétiés élastiques, ils expriment cependant ces coefficients analytiquement et donnent une expression compacte de l'énergie.

Dans cette note, les auteurs reconsidèrent $\propto$ même problème, mais dans les deux hypothèses de dislocations intrinsèques el extrinsèques. De plus, une solution entièrement analytique est donnée pour deux milieux à propriétés élastiques différentes. La démarche s'inspire d'un article paru dans $\propto$ journal (Bonnet 1981), traitant le cas de deux milieux semi-infinis, et dont les résultats ont été appliqués récemment pour décrire le champ des déplacements de dislocations intrinsèques à l'interface $\mathrm{GaSb} / \mathrm{GaAs}$, pour une épaisseur moyenne de $\mathrm{GaSb}$ variant de $h=4,2 \mathrm{~nm}$ à $30 \mathrm{~nm}$ (Bourret et al. 1991). Une solution plus appropriée devrait donner des expressions analytiques dépendantes de $h$. Nous nous proposons dans cette note de donner ces expressions et d'illustrer le résultat pour un réseau carré de dislocations purement coin à l'interface $\mathrm{GaSb} / \mathrm{GaAs}$ dont le désaccord paramétrique, ou Vernier, vaut $7 \%$ environ.

\$2. SOLUTION (II:NI:RAI.E: IDIS FOUATIONS IDE LELLASTICITE: PI.ANE CORRESPONDANT A UN CHAMP IDE DEFFORMATION PI:RIODIQUE LE LONG D'UNE INTERFACE SEPPARANT DEUX MILIEUX DIFFERENTS

Soit un milieu élastiquement isotrope. En combinant les èquations d'équilibre d'un élément de volume aux relations linéaires de Hooke, nous obtenons l'èquation diff́̉rentielle classique de l'élasticité, où $u_{k}$ représente les trois composantes du champ des déplacements $(i, k=1,2,3)$. En adoptant la convention de sommation d'Einstein et en notant par une virgule l'opération de dérivation, cette équation s'écrit

$$
(\lambda+u) u_{u_{u}}+u u_{u_{u}}=0 .
$$

$\lambda$ et $\mu$ sont les coefficients de Lamé du milicu déformé. Dans la suite du texte. une grandeur algébrique qui n'a pas un signe + ou - affecté en indice supćrieur est utilisable indifféremment pour chacun des milieux. Le coefficient de Poisson est v. 
La déformation est plane et supposéc strictement périodique le long de l'axe $O x_{1}$ (fig. 1) avec la période $\Lambda$. Le repère cartésien est centré sur le coeur d'une dislocation interfaciale. Posons $\omega=2 \pi / \Lambda$, et adoptons Re et Im comme symboles signifiant respectivement 'partie réelle de' et 'partie complexe de'. En admettant que le champ des déformations est périodique selon l'axe $\mathrm{Ox}_{1}$, il est facile de montrer que les composantes du champ des déplacements $u_{k}$ peuvent s'écrire sous l'une ou l'autre forme suivante (Bonnet 1981, éqn. (5)):

$$
\begin{aligned}
& u_{k}=\sum_{n=-\infty}^{\infty} U_{k}^{(n)} \exp \left(\operatorname{in\omega } x_{1}\right) \\
& u_{k}=u_{k}^{0}+v_{k 1}^{0} x_{1}+v_{k 2}^{0} x_{2}+\sum_{n=1}^{\infty}\left[2 \operatorname{Re}\left(U_{k}^{(n)}\right) \cos \left(n \omega x_{1}\right)-2 \operatorname{Im}\left(U_{k}^{(n)}\right) \sin \left(n \omega x_{1}\right)\right],
\end{aligned}
$$

oủ les termes de rang $n=0$, pour $k=1,2,3$, sont des lonctions linéaires réelles de $x_{1}$ et $x_{2}$, ef où les $U_{k}^{(n)}$ ne dépendent que de $x_{2}$.

En insérant l'eqn. (2 a) dans l'eqn. (1), nous obtenons, pour chaque harmonique de rang n non nul,

$$
\begin{aligned}
& (\lambda+2 \mu)\left(-n^{2} \omega^{2}\right) U_{1}+(\lambda+\mu)(\mathrm{i} n \omega) U_{2.2}+\mu U_{1.22}=0, \\
& \mu\left(-n^{2} \omega^{2}\right) U_{2}+(\lambda+\mu)(\mathrm{in} \omega) U_{1.2}+(\lambda+2 \mu) U_{2.22}=0, \\
& \left(-n^{2} \omega^{2}\right) U_{3}+U_{3.22}=0
\end{aligned}
$$

La solution générale du système $(3)+(4)+(5)$ est donnée par

$U_{1}=\left(P+O n \omega x_{3}\right) \exp \left(-n \omega x_{3}\right)+\left(R+S n \omega x_{3}\right) \exp \left(n \omega x_{3}\right)$.

$U_{2}=\mathrm{i}\left[P+Q(3-4 v)+Q n \omega x_{2}\right] \exp \left(-n \omega x_{2}\right)-\mathrm{i}\left[R-S(3-4 v)+S n \omega x_{2}\right] \exp \left(n \omega x_{2}\right)$,

$U_{3}=T \exp \left(n \omega x_{2}\right)+U \exp \left(n \omega x_{2}\right)$.

Les constantes complexes $P, Q, R, S, T$ et $C$ dépendent des conditions aux limites. Dans le cas limite de deux milieux semi-infinis, les constantes $R^{+}, S^{+}, P^{-}, Q^{-}, U^{+}$et $T^{-}$sont choisies nulles pour assurer la convergence du champ des déformations loin de l'interface (Bonnet 1981).

Léquation différentielle (1) n'implique aucune condition particulière sur les constantes $u_{k}^{0}, v_{k 1}^{0}, v_{k 2}^{0}$ qui ne dépendent donc que des conditions aux limites.

\section{§3. CRIttitris de CONVIRGienCE ET D'ÉQUILIBRE DES SOLUTIONS OBTENUES}

\subsection{Condition de convergence des solutions}

Nous admettons que dans le milieu $x_{2}<0$, l'énergie élastique stockée par unité de surface demeure linie, pour les deux types de dislocations intrinsèques et extrinsèques. Comme toutes les contraintes tendent vers zéro dans le milieu $x_{2}<0$ loin du joint $\left(\left|x_{2}\right| \rightarrow \infty\right)$, les constantes $v_{k 1}^{0}$ et $v_{k 2}^{0}$ sont nulles. Pour la méme raison, avec l'eqn. $(2 b)$ substituć dans l'eqns. (6)-(8), on a

$$
P^{-}=O^{-}=T^{-}=0
$$




\subsection{Condition d'équilibre d'un volume entourant une singularité de déformation (coeur d'une dislocation)}

Nous vérifions que la résultante et le moment des forces agissant sur un cylindre de matière renfermant une singularité de déformation est bien nulle pour la solution générale donnée ci-dessus. Puisque les conditions d'équilibre de la matière sont vérifiées dans le matériau en dehors du coeur, n'importe quel volume de matière contenant une singularité peut être choisi pour calculer cette résultante (voir par exemple Hirth et Lothe 1982). Le schéma de la fig. 1 représente un parallélépipéde rectangle $\mathrm{A} B C D E F$, en pointillés, qui entoure le coeur d'une dislocation. Ce volume est limité le long de $\mathrm{O} x_{3}$ par une longueur unité. Le long de $\mathrm{O} x_{1}$, les points $\mathrm{A}$ et $\mathrm{D}$ sont distants d'une période et sont situés à égale distance des coeurs des dislocations les plus proches. Le segment BC se trouve sur la surface libre. Les forces extérieures agissant sur $\mathrm{CE}$ et $\mathrm{BF}$, sur deux facettes de largeur $\mathrm{d} x_{2}$ placées le long d'une même ordonnée, sont exactement opposées puisque la longueur $\mathrm{AD}$ est égale à une période. Ces forces sont nulles le long de BC. Elles sont aussi nulles le long de EF lorsque $\propto$ segment se trouve infiniment loin de l'interfacc. Au total, la résultante des forces chcrchćc cst donc bicn nulle. Le moment de ces forces, pris par rapport au coeur de la dislocation placé entre $A$ et $D$, est aussi nul pour les mêmes raisons de symétrie.

\section{§4. Conditions aUX Limites CORRESPONDANT AUX Dislocations INTRINSÈQUeS}

\subsection{Condition en déplacement}

Nous admettons ici que l'interface est plane avec une seule famille de dislocations intrinsèques réparties périodiquement et que le champ des déplacements est strictement périodique (période 1 ). Cette dernière condition implique que $v_{k 1}^{0}=v_{k 2}^{0}=0$. Le champ des déplacements est discontinu le long de l'interface, avec une discontinuité variant linéairement à l'intérieur d'une période (Bonnet 1981).

Cette propriété exprime qu'à partir d'un état non déformé, obtenu en coupant les liaisons atomiques le long de l'interface, l'état final est obtenu en raccordant les plans réticulaires de façon que le déplacement relatif $u_{k}^{+}-u_{k}^{-}$soit périodique le long de $\mathrm{O} x_{1}$ et nul à mi-chemin entre deux coeurs de dislocations, en $\mathrm{A}$ sur la fig. 1. Cette hypothèse traduit, au niveau atomique, le rétablissement des unités structurales atomiques le long d'une facette interfaciale. Ainsi, une dizaine d'images à haute résolution de divers joints interphases comportant des singularités du champ des déplacements ont pu être interprétées a vec une bonne précision (Loubradou 1990, Bonnet, Loubradou, Catana et Stadelmann 1991, Pénisson 1991). Il est facile de montrer (Bonnet 1981) qu'au voisinage immédiat d'une dislocation intrinsèque, le champ des déplacements est très proche de celui d'une dislocation extrinsèque, orientée selon l'axe $\mathrm{O} x_{3}$, de vecteur de Burgers $b_{k}(k=1,2,3)$.

La linéarité du déplacement relatif peut être exprimée de trois façons (ici $x_{2}=0$ ):

$$
\begin{aligned}
& u_{k}^{+}-u_{k}^{-}=\left(\frac{b_{k}}{\Lambda}\right) x_{1}-\frac{b_{k}}{2}, \\
& u_{k}^{+}-u_{k}^{-}=\sum_{-}^{\infty} \frac{\mathrm{i} b_{k}}{2 \pi n} \exp \left(\mathrm{in} \omega x_{1}\right), \\
& u_{k}^{+}-u_{k}^{-}=\sum^{\infty} \frac{-b_{k}}{\pi n} \sin \left(n \omega x_{1}\right),
\end{aligned}
$$

oủ $n$ décrit l'ensemble des entiers relatifs non nuls dans l'éqn. (10b). 
La série (2a), appliquée aux deux milieux + et - et l'éqn. (10b) imposent donc que

$$
\begin{aligned}
U_{k}^{+}-U_{k}^{-} & =\frac{b_{k}}{2 n \pi}, \\
u_{k}^{0+} & =u_{k}^{0-}
\end{aligned}
$$

Le champ des déplacements est donc défini à un vecteur translation près, identique pour les milieux + et - . Ce vecteur, qui pcut être choisi de façon arbitraire, a des composantes que nous choisissons nulles pour simplifier $u_{k}^{0+}=u_{k}^{0-}=0$.

Le champ des déplacements $(2 b)$ s'écrit donc finalement

$$
u_{k}=2 \sum_{n=1}^{\infty}\left[\operatorname{Re}\left(U_{k}^{(n)} \cos \left(n \omega x_{1}\right)-\operatorname{Im}\left(U_{k}^{(n)}\right) \sin \left(n \omega x_{1}\right)\right]\right.
$$

\subsection{Condition en contraintes}

La continuité des forces au travers de l'interface impose que pour $x_{2}=0$

$$
\sigma_{\rightarrow 2}^{+}=\sigma_{, L}^{-}
$$

Explicitons maintenant cette condition en déterminant tout d'abord les expressions des déformations $(i, j=1,2,3)$

$$
\varepsilon_{i i}=\frac{1}{3}\left(u_{i}+u_{i}\right)
$$

A partir des relations de Hooke appliquées à la déformation plane

$$
\sigma_{11}=(\lambda+2 u) \varepsilon_{1,}+\lambda \varepsilon_{3,} \text { etc.. }
$$

et de l'expression du champ des déplacements, série (12), nous obtenons les délormations (non nulles)

$$
\begin{aligned}
& \varepsilon_{11}=\sum_{1}\left[-2 n \omega \operatorname{Im}\left(U_{1}\right) \cos \left(n \omega x_{1}\right)-2 n \omega \operatorname{Re}\left(U_{1}\right) \sin \left(n \omega x_{1}\right)\right], \\
& \varepsilon_{22}=\sum^{\infty}\left[2 \operatorname{Re}\left(U_{2,2}\right) \cos \left(n \omega x_{1}\right)-2 \operatorname{Im}\left(U_{2.2}\right) \sin \left(n \omega x_{1}\right)\right], \\
& \varepsilon_{12}=\sum_{1}\left\{\left[\operatorname{Re}\left(U_{1.2}\right)-n \omega \operatorname{Im}\left(U_{2}\right)\right] \cos \left(n \omega x_{1}\right)+\left[-\operatorname{Im}\left(U_{1.2}\right)-n \omega \operatorname{Re}\left(U_{2}\right)\right] \sin \left(n \omega x_{1}\right)\right\},
\end{aligned}
$$

$$
\varepsilon_{13}=\sum_{1}\left[-n \omega \operatorname{lm}\left(U_{3}\right) \cos \left(n \omega x_{1}\right)-n \omega \operatorname{Rc}\left(U_{3}\right) \sin \left(n \omega x_{1}\right)\right],
$$


$\varepsilon_{23}=\sum_{1}\left[\operatorname{Re}\left(U_{3,2}\right) \cos \left(n \omega x_{1}\right)-\operatorname{Im}\left(U_{3,2}\right) \sin \left(n \omega x_{1}\right)\right]$.

Les contraintes $\sigma_{2 k}$ (réelles) ont donc les expressions (complexes) suivantes:

$$
\begin{aligned}
& \sigma_{22}=\sum_{-\infty}^{\infty}\left[i \lambda n \omega U_{1}+(\lambda+2 \mu) U_{2,2}\right] \exp \left(\operatorname{in} \omega x_{1}\right) \\
& \left.\sigma_{21}=\sum_{-\infty} \mu\left(U_{1,2}+\mathrm{in} \omega U_{2}\right) \exp \ddot{i n} \omega x_{1}\right) \\
& \sigma_{23}=\sum_{-\infty} \mu U_{3,2} \exp \left(\operatorname{in} \omega x_{1}\right)
\end{aligned}
$$

ce qui donne, en termes réels

$$
\begin{aligned}
\sigma_{22}= & \sum_{1}\left\{\left[\lambda(-2 n \omega) \operatorname{Im}\left(U_{1}\right)+2(\lambda+2 \mu) \operatorname{Re}\left(U_{2,2}\right)\right] \cos \left(n \omega x_{1}\right)\right. \\
& \left.+\left[\lambda(-2 n \omega) \operatorname{Re}\left(U_{1}\right)-2(\lambda+2 \mu) \operatorname{Im}\left(U_{2,2}\right)\right] \sin \left(n \omega x_{1}\right)\right\}, \\
\sigma_{21}= & \sum\left\{2 \mu\left[\operatorname{Re}\left(U_{1,2}\right)-n \omega \operatorname{lm}\left(U_{2}\right)\right] \cos \left(n \omega x_{1}\right)\right. \\
& \left.+2 \mu\left\lceil-\operatorname{Im}\left(U_{1,2}\right)-n \omega \operatorname{Re}\left(U_{2}\right)\right] \sin \left(n \omega x_{1}\right)\right\}, \\
\sigma_{23}= & \sum\left[2 \mu \operatorname{Re}\left(U_{3,2}\right) \cos \left(n \omega x_{1}\right)-2 \mu \operatorname{Im}\left(U_{3,2}\right) \sin \left(n \omega x_{1}\right)\right] .
\end{aligned}
$$

Dans ces expressions, les dérivées $U_{1,2}$ et $U_{2,2}$ et $U_{3,2}$, facilement calculables, sont les suivantes

$$
\begin{aligned}
U_{1,2}= & {\left[Q n \omega-n \omega\left(P+Q n \omega x_{2}\right)\right] \exp \left(-n \omega x_{2}\right)+\left[S n \omega+n \omega\left(R+S n \omega x_{2}\right)\right] \exp \left(n \omega x_{2}\right), } \\
U_{2,2}= & -i n \omega\left[P+Q(2-4 v)+Q n \omega x_{2}\right] \exp \left(-n \omega x_{2}\right)-i n \omega[R-S(2-4 v) \\
& \left.+S n \omega x_{2}\right] \exp \left(n \omega x_{2}\right), \\
U_{2,3}= & -n \omega T \exp \left(-n \omega x_{2}\right)-n \omega U \exp \left(n \omega x_{2}\right) .
\end{aligned}
$$

La condition (13), combinće a vec l'éqns. (21a), (21 b) et (21 c) implique donc les trois relations suivantes, dans lesquelles il faut faire $x_{2}=0$ :

$$
\begin{aligned}
& \left\lceil\mathrm{i} \lambda^{+} n \omega U_{1}^{+}+\left(\lambda^{+}+2 u^{+}\right) U_{\lrcorner}^{+},\right\rceil-\left\lceil i \lambda^{-} n \omega U_{-}^{-}+\left(\lambda^{-}+2 u^{-}\right) U_{\curvearrowright}^{-},\right\rceil=0 . \quad(24 a
\end{aligned}
$$

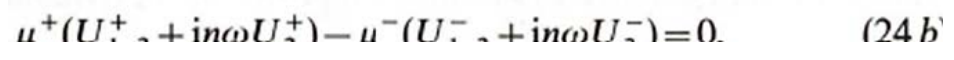

$$
\begin{aligned}
& u^{+} U_{2}^{+},-u^{-} U_{2},=0 . \quad\left(24 c^{\prime}\right.
\end{aligned}
$$


4.3. Condition aux limites à la surface libre $\left(x_{2}=h\right)$

Les contraintes de surface sont nulles à la surface. Cette condition est exprimée par les trois équations suivantes, pour lesquelles $x_{2}=h$ :

$$
\begin{aligned}
\mathrm{i} \lambda^{+} n \omega U_{1}^{+}+\left(\lambda^{+}+2 \mu^{+}\right) U_{2.2}^{+} & =0, \\
U_{1,2}^{+}+\mathrm{in} \omega U_{2}^{+} & =0, \\
U_{3,2}^{+} & =0 .
\end{aligned}
$$

\section{§5. DÉTERMINATION EXPLICITE DU CHAMP DES DÉPLACEMENTS ET DES CONTRAINTES DES DISLOCATIONS INTRINSĖQUES}

\subsection{Détermination des constantes complexes}

Pour trouver explicitement chacun des coefficients de Fourier $U_{k}^{(n)}$ du champ des déplacements (12), il faut résoudre analytiquement les éqns. (11), (24) et (25). Pour le milieu $x_{2}<0$, les conditions (9) s'appliquent. Il demeure donc à résoudre un système linéaire de neuf́ équations à neuf inconnues en

$$
P^{+}, Q^{+}, R^{+}, S^{+}, T^{+}, U^{+}, R^{-}, S^{-} \text {et } U^{-} \text {. }
$$

Ce système se découple en fait en deux sous-systèmes indépendants. Le premier comporte seulement les inconnues $T^{+}, U^{+}$et $U^{-}$. Il s'écrit

$$
\begin{aligned}
T^{+}+U^{+}-U^{-} & =\frac{\mathrm{i} b_{3}}{2 n \pi}, \\
\mu^{+}\left(-T^{+}+U^{+}\right)-\mu^{-} U^{-} & =0 \\
-T^{+}+U^{+} \exp (2 n \omega h) & =0 .
\end{aligned}
$$

Les solutions sont purement complexes. En notant $k=\mu^{+} / \mu^{-}$, elles s'écrivent

$$
\begin{aligned}
U^{+} & =\frac{\mathrm{i} b_{3}}{2 n \pi 1-k+(1+k) \exp (2 n \omega h)^{\prime}} \\
T^{+}=U^{+} \exp (2 n \omega h)= & \begin{array}{l}
\mathrm{i} b_{3} \quad \frac{\exp (2 n \omega h)}{2 n \pi} 1-k+\frac{(1+k) \exp (2 n \omega h)^{\prime}}{2 n} \\
U^{-}=k\left(-T^{+}+U^{+}\right) \\
\mathrm{i} b_{3} \quad \frac{k(1-\exp (2 h n \omega))}{2 n \pi 1-k+(1+k) \exp (2 h n \omega)}
\end{array}
\end{aligned}
$$

La composante du champ des déplacement parallc̀lement aux dislocations ne dépend donc que de la composante vis du vecteur de Burgers b et du rapport $k$ des modules de cisaillement.

L'autre système d'équations comporte les six inconnues $P^{+}, Q^{+}, R^{+}, S^{+}, R^{-}$et $S^{-}$. Il s'écrit sous la forme d'un produit matriciel, où la matrice colonne $X_{j}(j=1-6)$ renierme ces six inconnues complexes écrites dans le même ordre de haut en bas:

$$
\mathbf{A} X=\mathbf{B} \text {. }
$$

Dans cette équation, les matrices $\mathbf{A}$ et $\mathbf{B}$ sont respectivement une matrice carrée réelle $6 \times 6$ et une matricc cononne. En posant $E=\exp (n \omega h)$, l'éqn. (30) s'écrit aussi l'égn. (30a).

Ainsi, la seule quantité complexe intervenant dans cette équation est le premier élément de la matrice $B$. Cette équation a été résolue analytiquement en utilisant le 


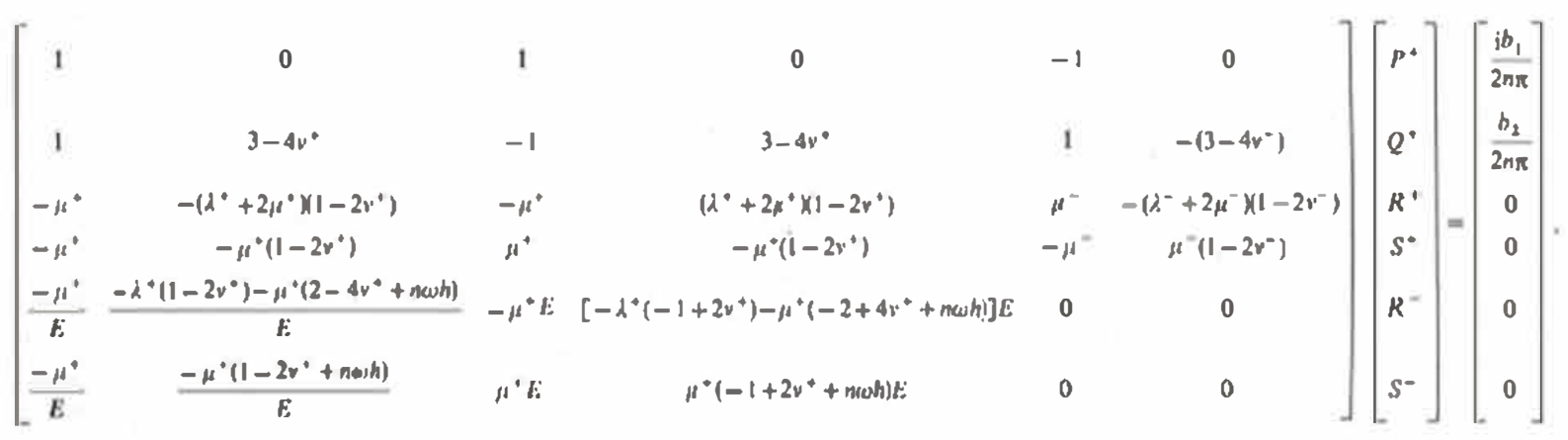


logiciel de calcul formel Mathematica. Le résultat peut se mettre sous la forme suivante:

$X_{j} \quad \frac{\left[t_{j 1}+t_{j 2} \exp (2 h n \omega)+t_{j 3} \exp (4 h n \omega)\right] b_{2}+\left[i_{j 4}+t_{j 5} \exp (2 h n \omega)+t_{j 6} \exp (4 h n \omega)\right] i b_{1}}{2 n \pi \Delta}$,

$\Delta=\alpha+\beta \exp (2 h n \omega)+\gamma \exp (4 h n \omega)$.

Les termes $t_{j m}(j, m=1-6)$ et $\alpha, \beta$ et $\gamma$ sont explicités dans l'Annexe en fonction de $k$, du produit $h n \omega$ et des coefficients de Poisson $v^{+}$et $v^{-}$. La solution $(31 a)$ a été vérifice en calculant de deux façons les solutions $X_{j}$ : d'une part en inversant numériquement l'ćqn. (30), pour quelques harmoniques de rang $n$; dautre part en utilisant les expressions analytiques des éléments $t_{i j}$ (Annexe). L'expression ( $\left.31 a\right)$ est obtenue en simplifiant une fraction par le produit $\left(-1-3 k+4 k v^{-}\right)\left(3+k-4 v^{+}\right)$. Ce produit est toujours différent de zéro comme il est facile de le vérifier, puisque chacun des coefficients de Poisson est compris entre 0 et $\frac{1}{2}$ et que $k$ est toujours positif (il suffit que $-1 \leqslant v^{ \pm} \leqslant \frac{1}{2}$ ). Lorsque $h n \omega$ devient suffisamment grand (c'est-à-dire, pour $n$ tendant vers l'infini), les solutions ont des comportements différents: $n S^{+}$et $n R^{+}$tendent vers zéro avec un terme $\exp (-2 n \omega h)$, tandis que $n P^{+}, n Q^{+}, n R^{-}$et $n S^{-}$tendent vers des constantes

$$
\begin{aligned}
& \left(n P^{+}\right)_{\lim } \begin{array}{c}
2 k\left(2-3 v^{-}-3 v^{+}+4 v^{-} v^{+}\right) b_{2}-\left(3+5 k-6 k v^{-}-4 v^{+}-6 k v^{+}+8 k v^{-} v^{+}\right) \mathrm{i} b_{1} \\
\left.2 \jmath d-1-3 k+4 k v^{-}\right)\left(3+k-4 v^{+}\right)
\end{array} \\
& \left(n Q^{+}\right)_{\lim }=\frac{b_{2}-\mathrm{i} b_{1}}{2 \pi\left(3+k-4 v^{+}\right)^{\prime}} \\
& \left(n R^{-}\right)_{1: \ldots} \frac{-2 k\left(2-3 v^{-}-3 v^{+}+4 v^{-} v^{+}\right) b_{2}-k\left(-5-3 k+6 v^{-}+4 k v^{-}+6 v^{+}-8 v^{-} v^{+}\right) \mathrm{i} b_{1}}{2 \pi\left(-1-3 k+4 k v^{-}\right)\left(3+k-4 v^{+}\right)} \\
& \left(n S^{-}\right)_{\lim }=\frac{k\left(b_{2}+\mathrm{i} b_{1}\right)}{2 \pi\left(-1-3 k+4 k v^{-}\right)} .
\end{aligned}
$$

La convergence du champ des déplacements (12) est donc lente puisque en $1 / n$. Ce résultat est conforme aux propriétćs des sćries de Fourier de fonctions discontinues (Bass 1964).

5.2. Expression analytigue du champ des déplacements

En insérant dans les éqns. (6-8) et (12) les expressions des solutions trouvées cidessus, nous obtenons les expressions explicites du champ des déplacements pour les deux milieux:

$$
\begin{aligned}
u_{1}^{+}= & \sum_{1}^{\infty}\left(b_{2}\left\{\left(t_{12}+n \omega t_{22} x_{2}\right) \exp \left[n \omega\left(2 h-x_{2}\right)\right]+t_{13}+n \omega t_{23} x_{2}\right) \exp \left[n \omega\left(4 h-x_{2}\right)\right]\right. \\
& \left.+\left(t_{31}+n \omega t_{41} x_{2}\right) \exp \left(n \omega x_{2}\right)+\left(t_{32}+n \omega t_{42} x_{2}\right) \exp \left[n \omega\left(2 h+x_{2}\right)\right]\right\} \cos \left(n \omega x_{1}\right) \\
& +b_{1}\left\{\left(-t_{15}-n \omega t_{25} x_{2}\right) \exp \left[n \omega\left(2 h-x_{2}\right)\right]+\left(-t_{16}-n \omega t_{26} x_{2}\right) \exp \left[n \omega\left(4 h-x_{2}\right)\right]\right. \\
& \left.\left.+\left(-t_{34}-n \omega t_{44} x_{2}\right) \exp \left(n \omega x_{2}\right)+\left(-t_{35}-n \omega t_{45} x_{2}\right) \exp \left[n \omega\left(2 h+x_{2}\right)\right]\right\} \frac{\sin \left(n \omega x_{1}\right)}{n \pi \Delta}\right),
\end{aligned}
$$




$$
\begin{aligned}
& u_{2}^{+}=\sum_{1}^{\infty}\left(b _ { 1 } \left\{\left[-t_{15}+t_{25}\left(-3+4 v^{+}-n \omega x_{2}\right)\right] \exp \left[n \omega\left(2 h-x_{2}\right)\right]\right.\right. \\
& \times\left[-t_{16}+t_{26}\left(-3+4 v^{+}-n \omega x_{2}\right)\right] \exp \left[n \omega\left(4 h-x_{2}\right)\right] \\
& +\left[t_{34}+t_{44}\left(-3+4 v^{+}+n \omega x_{2}\right)\right] \exp \left(n \omega x_{2}\right) \\
& \left.+\left[t_{35}+t_{45}\left(-3+4 v^{+}+n \omega x_{2}\right)\right] \exp \left[n \omega\left(2 h+x_{2}\right)\right]\right\} \frac{\cos \left(n \omega x_{1}\right)}{n \pi \Delta} \\
& +b_{2}\left\{\left[-t_{12}+t_{22}\left(-3+4 v^{+}-n \omega x_{2}\right)\right] \exp \left[n \omega\left(2 h-x_{2}\right)\right]\right. \\
& +\left[-t_{13}+t_{23}\left(-3+4 v^{+}-n \omega x_{2}\right)\right] \exp \left[n \omega\left(4 h-x_{2}\right)\right] \\
& +\left[t_{31}+t_{41}\left(-3+4 v^{+}+n \omega x_{2}\right)\right] \exp \left(n \omega x_{2}\right) \\
& \left.\left.+\left[t_{32}+t_{42}\left(-3+4 v^{+}+n \omega x_{2}\right)\right] \exp \left[n \omega\left(2 h+x_{2}\right)\right]\right\} \frac{\sin \left(n \omega x_{1}\right)}{n \pi \Delta}\right), \\
& u_{3}^{+}=\frac{-b_{3}}{\pi} \sum_{1}^{\infty} \frac{\exp \left[n \omega\left(x_{2}-2 h\right)\right]+\exp \left(-n \omega x_{2}\right)}{n\{1+k+(1-k) \exp [-2 n \omega h]\}} \sin \left(n \omega x_{1}\right), \\
& u_{1}^{-}=\sum_{1}^{\infty}\left(b _ { 2 } \left\{\left(t_{51}+n \omega t_{61} x_{2}\right) \exp \left(n \omega x_{2}\right)+\left(t_{52}+n \omega t_{62} x_{2}\right) \exp \left[n \omega\left(2 h+x_{2}\right)\right]\right.\right. \\
& \left.+\left(t_{53}+n \omega t_{63} x_{2}\right) \exp \left[n \omega\left(4 h+x_{2}\right)\right]\right\} \frac{\cos \left(n \omega x_{1}\right)}{n \pi \Delta} \\
& +b_{1}\left[\left(-t_{54}-n \omega t_{64} x_{2}\right) \exp \left(n \omega x_{2}\right)+\left(-t_{55}-n \omega t_{65} x_{2}\right) \exp \left[n \omega\left(2 h+x_{2}\right)\right]\right. \\
& \left.+\left(-t_{56}-n \omega t_{66} x_{2}\right) \exp \left[n \omega\left(4 h+x_{2}\right)\right] \frac{\sin \left(n \omega x_{1}\right)}{n \pi \Delta}\right) \text {, } \\
& u_{2}^{-}=\sum_{1}^{\infty}\left(b _ { 1 } \left\{\left[t_{54}+t_{64}\left(-3+4 v^{-}+n \omega x_{2}\right)\right] \exp \left(n \omega x_{2}\right)\right.\right. \\
& +\left[t_{55}+t_{65}\left(-3+4 v^{-}+n \omega x_{2}\right)\right] \exp \left[n \omega\left(2 h+x_{2}\right)\right] \\
& \left.+\left[t_{56}+t_{66}\left(-3+4 v^{-}+n \omega x_{2}\right)\right] \exp \left[n \omega\left(4 h+x_{2}\right)\right]\right\} \frac{\cos \left(n \omega x_{1}\right)}{n \pi \Delta} \\
& +b_{2}\left\{\left[t_{51}+t_{61}\left(-3+4 v^{-}+n \omega x_{2}\right)\right] \exp \left(n \omega x_{2}\right)\right. \\
& +\left[t_{52}+t_{62}\left(-3+4 v^{-}+n \omega x_{2}\right)\right] \exp \left[n \omega\left(2 h+x_{2}\right)\right] \\
& \left.\left.+\left[t_{53}+t_{63}\left(-3+4 v^{-}+n \omega x_{2}\right)\right] \times \exp \left[n \omega\left(4 h+x_{2}\right)\right]\right\} \frac{\sin \left(n \omega x_{1}\right)}{n \pi \Delta}\right), \\
& u_{3}^{-}=\frac{-b_{3}}{\pi} k \sum_{1}^{\infty} \frac{\exp \left[n \omega\left(x_{2}-2 h\right)\right]-\exp \left(n \omega x_{2}\right)}{n[1+k+(1-k) \exp (-2 n \omega h)]} \sin \left(n \omega x_{1}\right) .
\end{aligned}
$$

5.3. Expression du champ des contraintes et de la dilatation volumique Les expressions analytiques des contraintes sont obtenues en appliquant les éqns. (16)-(20) puis l'eqn. (15). Les contraintes $\sigma_{33}$ sont calculées par la relation classique $\sigma_{33}=v\left(\sigma_{11}+\sigma_{22}\right)$. 
(a) Milicu $x_{2}>0$,

$$
\begin{aligned}
\sigma_{1:}^{+}= & 2 \omega \mu^{+} \sum_{1}^{\infty}\left(b _ { 1 } \left\{\left[-t_{15}+t_{25}\left(2 v^{+}-n \omega x_{2}\right)\right] \exp \left[n \omega\left(2 h-x_{2}\right)\right]\right.\right. \\
& +\left[-t_{16}+t_{26}\left(2 v^{+}-n \omega x_{2}\right)\right] \exp \left[n \omega\left(4 h-x_{2}\right)\right] \\
& +\left[-t_{34}+t_{44}\left(-2 v^{+}-n \omega x_{2}\right)\right] \exp \left(n \omega x_{2}\right)+\left[-t_{35}+t_{45}\left(-2 v^{+}-n \omega x_{2}\right)\right] \\
& \left.\times \exp \left[n \omega\left(2 h+x_{2}\right)\right]\right\} \cos \left(n \omega x_{1}\right) \\
& +b_{2}\left\{\left[-t_{12}+t_{22}\left(2 v^{+}-n \omega x_{2}\right)\right] \exp \left(n \omega\left(2 h-x_{2}\right)\right]\right. \\
& +\left[-t_{13}+t_{23}\left(2 v^{+}-n \omega x_{2}\right)\right] \exp \left[n \omega\left(4 h-x_{2}\right)\right] \\
& +\left[-t_{31}+t_{41}\left(-2 v^{+}-n \omega x_{2}\right)\right] \exp \left(n \omega x_{2}\right)+\left[-t_{32}+t_{42}\left(-2 v^{+}-n \omega x_{2}\right)\right] \\
& \left.\left.\times \exp \left[n \omega\left(2 h+x_{2}\right)\right]\right\} \sin \left(n \omega x_{1}\right)\right),
\end{aligned}
$$

$$
\begin{aligned}
\sigma_{22}^{+}= & 2 \omega \mu^{+} \sum_{1}^{\infty}\left(b _ { 1 } \left\{\left[t_{15}+t_{25}\left(2-2 v^{+}+n \omega x_{2}\right)\right] \exp \left[n \omega\left(2 h-x_{2}\right)\right]\right.\right. \\
& +\left[t_{16}+t_{26}\left(2-2 v^{+}+n \omega x_{2}\right)\right] \exp \left[n \omega\left(4 h-x_{2}\right)\right] \\
& +\left[t_{34}+t_{44}\left(-2+2 v^{+}+n \omega x_{2}\right)\right] \exp \left(n \omega x_{2}\right) \\
& \left.+\left[t_{35}+t_{45}\left(-2+2 v^{+}+n \omega x_{2}\right)\right] \exp \left(n \omega\left(2 h+x_{2}\right)\right]\right\} \frac{\cos \left(n \omega x_{1}\right)}{\pi \Lambda} \\
& +b_{2}\left\{\left[t_{12}+t_{22}\left(2-2 v^{+}+n \omega x_{2}\right)\right] \exp \left[n \omega\left(2 h-x_{2}\right)\right]\right. \\
& +\left[t_{13}+t_{23}\left(2-2 v^{+}+n \omega x_{2}\right)\right] \exp \left[n \omega\left(4 h-x_{2}\right)\right] \\
& +\left[t_{31}+t_{41}\left(-2+2 v^{+}+n \omega x_{2}\right)\right] \exp \left(n \omega x_{2}\right) \\
& \left.\left.+\left[t_{32}+t_{42}\left(-2+2 v^{+}+n \omega x_{2}\right)\right] \exp \left[n \omega\left(2 h+x_{2}\right)\right]\right\} \frac{\sin \left(n \omega x_{1}\right)}{\pi \Delta}\right)
\end{aligned}
$$

$$
\begin{aligned}
\sigma_{21}^{+}= & 2 \omega \mu^{+} \sum_{1}^{\infty}\left(b _ { 2 } \left\{\left[-t_{12}+t_{22}\left(-1+2 v^{+}-n \omega x_{2}\right)\right] \exp \left[n \omega\left(2 h-x_{2}\right)\right]\right.\right. \\
& \left.+\left[-t_{13}+t_{23}\left(-1+2 v^{+}-n \omega x_{2}\right)\right] \exp n \omega\left(4 h-x_{2}\right)\right] \\
& +\left[t_{31}+t_{41}\left(-1+2 v^{+}+n \omega x_{2}\right)\right] \exp \left(n \omega x_{2}\right) \\
& \left.+\left[t_{32}+t_{42}\left(-1+2 v^{+}+n \omega x_{2}\right)\right] \exp \left[n \omega\left(2 h+x_{2}\right)\right]\right\} \frac{\cos \left(n \omega x_{1}\right)}{\pi \Delta} \\
& +b_{1}\left\{\left[t_{15}+t_{25}\left(1-2 v^{+}+n \omega x_{2}\right)\right] \exp \left[n \omega\left(2 h-x_{2}\right)\right]\right. \\
& +\left[t_{16}+t_{26}\left(1-2 v^{+}+n \omega x_{2}\right)\right] \exp \left[n \omega\left(4 h-x_{2}\right)\right] \\
& +\left[-t_{34}+t_{44}\left(1-2 v^{+}-n \omega x_{2}\right)\right] \exp \left(n \omega x_{2}\right) \\
& \left.\left.+\left[-t_{35}+t_{45}\left(1-2 v^{+}-n \omega x_{2}\right)\right] \exp \left[n \omega\left(2 h+x_{2}\right)\right]\right\} \sin \left(n \omega x_{1}\right)\right),
\end{aligned}
$$




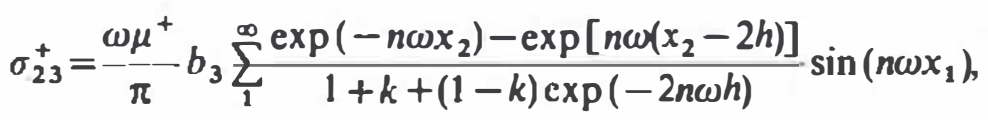

$$
\begin{aligned}
& \sigma_{13}^{+}=\frac{-\omega \mu^{+}}{\pi} b_{3} \sum_{1}^{\infty} \frac{\exp \left(-n \omega x_{2}\right)+\exp \left[n \omega\left(x_{2}-2 h\right)\right]}{1+k+(1-k) \exp (-2 n \omega h)} \cos \left(n \omega x_{1}\right) \text {. }
\end{aligned}
$$

Willis et al. (1990) ont aussi donnè l'éqn. (46), à une petit e erreur d'homogénéité près. En effet, leur éqn. (A 25) doit être divisée par la période $A$.

(b) Milieu $x_{2}<0$,

$$
\begin{aligned}
& \sigma_{11}^{-}=2 \omega \mu^{-} \sum_{1}^{\infty}\left(b _ { 1 } \left\{\left[-t_{54}+t_{64}\left(-2 v^{-}-n \omega x_{2}\right)\right] \exp \left(n \omega x_{2}\right)\right.\right. \\
& +\left[-t_{s s}+t_{6 s}\left(-2 v^{-}-n \omega x_{2}\right)\right] \exp \left[n \omega\left(2 h+x_{2}\right)\right] \\
& \left.+\left[-t_{56}+t_{66}\left(-2 v^{-}-n \omega x_{2}\right)\right] \exp \left[n \omega\left(4 h+x_{2}\right)\right]\right\} \frac{\cos \left(n \omega x_{1}\right)}{\pi A} \\
& +b_{2}\left\{\left[-t_{31}+t_{61}\left(-2 v^{-}-n \omega x_{2}\right)\right] \exp \left(n \omega x_{2}\right)\right. \\
& +\left[-t_{s 2}+t_{63}\left(-2 v^{-}-n \omega x_{2}\right)\right] \exp \left[n \omega\left(2 h+x_{2}\right)\right] \\
& \left.\left.+\left[-t_{53}+t_{63}\left(-2 v^{-}-n \omega x_{2}\right)\right] \exp \left[n \omega\left(4 h+x_{2}\right)\right]\right\} \frac{\sin \left(n \omega x_{1}\right)}{\pi \Delta}\right) \text {, } \\
& \sigma_{22}^{-}=2 \omega \mu^{-} \sum_{1}^{\infty}\left(b_{1}\left\{\left[t_{34}+l_{64}\left(-2+2 v^{-}+n \omega\right) x_{2}\right)\right] \exp \left(n \omega x_{2}\right)\right. \\
& +\left[t_{35}+t_{65}\left(-2+2 v^{-}+n \omega x_{2}\right)\right] \operatorname{cxp}\left[n \omega\left(2 h+x_{2}\right)\right] \\
& \left.+\left[t_{36}+t_{60}\left(-2+2 v^{-}+n \omega x_{2}\right)\right] \exp \left(n \omega\left(4 h+x_{2}\right)\right]\right\} \frac{\cos \left(n \omega x_{1}\right)}{\pi \Delta} \\
& +b_{2}\left\{\left[\iota_{51}+\iota_{61}\left(-2+2 v^{-}+n \omega x_{2}\right)\right] \exp \left(n \omega x_{2}\right)\right. \\
& +\left[t_{s 2}+\iota_{62}\left(-2+2 v^{-}+n \omega x_{2}\right)\right] \exp \left[n \omega\left(2 h+x_{2}\right)\right] \\
& \left.\left.+\left[t_{53}+\iota_{63}\left(-2+2 v^{-}+n \omega x_{2}\right)\right] \exp \left[n \omega\left(4 h+x_{2}\right)\right]\right\} \frac{\sin \left(n \omega x_{1}\right)}{\pi \Delta}\right) \text {, } \\
& \sigma_{21}^{-}=2 \omega \mu^{-} \sum_{1}^{\infty}\left(b _ { 2 } \left\{\left[t_{31}+t_{61}\left(-1+2 v^{-}+n \omega x_{2}\right)\right] \exp \left(n \omega x_{2}\right)\right.\right. \\
& +\left[t_{32}+t_{62}\left(-1+2 v^{-}+n \omega x_{2}\right)\right] \exp \left[n \omega\left(2 h+x_{2}\right)\right] \\
& \left.+\left[\iota_{53}+\imath_{63}\left(-1+2 v^{-}+n \omega x_{2}\right)\right] \exp \left[n \omega\left(4 h+x_{2}\right)\right]\right\} \frac{\cos \left(n \omega x_{1}\right)}{\pi \Delta} \\
& +b_{1}\left\{\left[-t_{34}+t_{64}\left(1-2 v^{-}-n \omega x_{2}\right)\right] \exp \left(n \omega x_{2}\right)\right. \\
& +\left[-l_{s s}+l_{6 s}\left(1-2 v^{-}-n \omega x_{2}\right)\right] \exp \left[n \omega\left(2 h+x_{2}\right)\right] \\
& \left.\left.+\left[-t_{36}+t_{66}\left(1-2 v^{-}-n \omega x_{2}\right)\right] \exp \left[n \omega\left(4 h+x_{2}\right)\right]\right\} \frac{\sin \left(n \omega x_{1}\right)}{\pi \Delta}\right) \text {, } \\
& \sigma_{23}^{-}=\frac{k \omega \mu^{-}}{\pi} b_{3} \sum_{1}^{\infty} \frac{(1-\exp (-2 n \omega h)) \exp \left(n \omega x_{2}\right)}{1+k+(1-k) \exp (-2 n \omega h)} \sin \left(n \omega x_{1}\right) \text {, }
\end{aligned}
$$




$$
\frac{-\omega \mu^{-}}{\pi} b_{3} \sum_{1}^{\infty} \frac{-\exp \left(n \omega x_{2}\right)+\exp \left[n \omega\left(x_{2}-2 h\right)\right]}{1+k+(1-k) \exp i-2 n \omega h)} \cos \left(n \omega x_{1}\right)
$$

La validité de ces expressions a été testée en vérifiant numériquement les conditions (13) et (25).

Pour les systèmes couche épitaxique/substrat, il est utile d'avoir une idée des variations de la dilatation volumique $\theta=\varepsilon_{11}+\varepsilon_{22}$ qui contrôle les flux de diffusion des espèces atomiques:

$$
\begin{aligned}
\theta^{+} & \frac{2 \omega\left(1-2 v^{+}\right)}{\pi} \sum_{1}^{\infty}\left(b _ { 1 } \left\{t_{25} \exp \left[n \omega\left(2 h-x_{2}\right)\right]+i_{26} \exp \left[n \omega\left(4 h-x_{2}\right)\right]-t_{44}\right.\right. \\
& \left.\times \exp \left(n \omega x_{2}\right)-t_{45} \exp \left[n \omega\left(2 h+x_{2}\right)\right]\right\} \cos \left(n \omega x_{1}\right) \\
& +b_{2}\left[t_{22} \exp \left[n \omega\left(2 h-x_{2}\right)\right]+t_{23} \exp \left[n \omega\left(4 h-x_{2}\right)\right]-t_{41}\right. \\
& \left.\left.\times \exp \left(n \omega x_{2}\right)-t_{42} \exp \left[n \omega\left(2 h+x_{2}\right)\right]\right] \sin \left(n \omega x_{1}\right)\right) \\
\theta^{-}= & \frac{2 \omega\left(-1+2 v^{-}\right)}{\pi} \sum_{1}^{\infty}\left(b _ { 1 } \left\{t_{64} \exp \left(n \omega x_{2}\right)+t_{65} \exp \left[n \omega\left(2 h+x_{2}\right)\right]+t_{66}\right.\right. \\
& \left.\times \exp \left[n \omega\left(4 h+x_{2}\right)\right]\right\} \frac{\cos \left(n \omega x_{1}\right)}{\Lambda} \\
& +b_{2}\left\{t_{61} \exp \left(n \omega x_{2}\right)+t_{62} \exp \left[n \omega\left(2 h+x_{2}\right)\right]+t_{63}\right. \\
& \left.\left.\times \exp \left[n \omega\left(4 h+x_{2}\right)\right]\right\} \frac{\sin \left(n \omega x_{1}\right)}{\Delta}\right) .
\end{aligned}
$$

\section{§6. Condition aUX limites CORRFSpondant aUX Disl.ocations fXtrinsèeufs}

Les dislocations interfaciales, dites 'extrinsèques', sont des dislocations de translation (Friedel 1979). Le champ élastique de telles dislocations à l'interface couche épitaxique/substrat a récemment été étudié par Willis et al. (1990). Ces auteurs les introduisent par une déformation plastique du substrat, préalablement déformé élastiquement de façon homogène. Cette déformation plastique est engendrée par des glissements périodiques, depuis la surface libre du substrat, d'un type déterminé de dislocation de translation. Les auteurs parviennent à donner une solution explicite lorsque les deux milieux ont les mêmes propriétés élastiques, et que les dislocations sont purement vis. Pour les dislocations coin, ils donnent une solution en séries de Fourier qui nécessite néanmoins l'inversion numérique d'une matrice complexe $4 \times 4$, pour chaque harmonique de la série.

Dans ce paragraphe, des solutions explicites applicables à deux milieux diffërents sont présentées.

\subsection{Condition en déplacement le long de l'interface}

Dans ce travail, les dislocations extrinsèques sont introduites diffëremment de Willis et al., par des glissements successifs le long de l'interface. La conséquence mathématique est décrite par l'éqn. (40) de Bonnet (1981). Elle exprime simplement que, le long de l'interface, les trois composantes de $u_{k}^{+}-u_{k}^{-}$, doivent varier par paliers, selon une courbe brisée en escalier (voir la fig. $3(b)$ de la référence ci-dessus). D'autre part, 
les composantes $u_{k}^{+}-u_{k}^{-}$ont des expressions générales imposées par l'éqn. $(2 b)$. Les conséquences sont donc les suivantes.

(1) Les coefficients $U_{k}^{(n)}$ de rang $n$ non nul, équa. $(2 a, b)$ sont les mêmes que pour les dislocations intrinseques. Il s'ensuit que tous les termes harmoniques sont identiques pour les dislocations intrinsèques et extrinsèques. Les deux champs ne se distinguent l'un de l'autre que par des termes linéaires en $x_{1}$ et $x_{2}$.

(2) Les relations suivantes doivent être vérifiées:

$$
\begin{aligned}
& u_{k}^{0+}-u_{k}^{0-}=-\frac{b_{k}}{2}, \\
& v_{k 1}^{0+}-v_{k 2}^{0-}=-\frac{b_{k}}{\Lambda},
\end{aligned}
$$

A l'éqn. (53a), il est possible d'ajouter une condition supplémentaire (arbitraire) pour définir l'état de translation global des deux milieux, par exemple

$$
\begin{aligned}
& u_{k}^{0-}=0, \\
& u_{k}^{0+}=-\frac{b_{k}}{2},
\end{aligned}
$$

Par contre, pour déterminer les six constantes $v_{k j}^{0}=0(j=1,2)$, il faut expliciter les conditions aux limites en contraintes.

\subsection{Condition en contraintes}

\subsubsection{Le long de l'interface}

En dérivant l'éqn. $(2 b)$ et en appliquant la relation de Hooke, il s'ensuit que le champ des dislocations extrinsèques est égal à celui des dislocations intrinsèques auquel il faut ajouter un champ des contraintes constant $\sigma_{i j}^{0}$ dont l'expression est

$$
\begin{aligned}
& \sigma_{11}^{0}=(\lambda+2 \mu) v_{11}^{0}+\lambda v_{22}^{0}, \\
& \sigma_{22}^{0}=\lambda v_{11}^{0}+(\lambda+2 \mu) v_{22}^{0}, \\
& \sigma_{33}^{0}=\lambda v_{11}^{0}+\lambda v_{22}^{0}, \\
& \sigma_{23}^{0}=\mu v_{32}^{0}, \\
& \sigma_{31}^{0}=\mu v_{31}^{0}, \\
& \sigma_{12}^{0}=\mu v_{12}^{0}+\mu v_{21}^{0} .
\end{aligned}
$$

La continuité des contraintes à l'interface exige donc les trois conditions suivants:

$$
\begin{aligned}
\lambda^{+} v_{11}^{0}+\left(\lambda^{+}+2 \mu^{+}\right) v_{22}^{0+} & =\lambda^{-} v_{11}^{0-}+\left(\lambda^{-}+2 \mu^{-}\right) v_{22}^{0}, \\
\mu^{+} v_{32}^{0+} & =\mu^{-} v_{32}^{0-}, \\
\mu^{+} v_{12}^{0+}+\mu^{+} v_{21}^{0+} & =\mu^{-} v_{22}^{0-}+\mu^{-} v_{21}^{0-} .
\end{aligned}
$$

\subsubsection{Dans le substrat, très loin de l'interface}

Nous admettons qu'il n'y a pas de champ de contraintes à longue distance dans le substrat. En effet, si ce champ était non nul, il engendrerait une énergie élastique stockée infinie, ce qui n'a pas de sens physique. Il s'ensuit donc que $(k=1,2,3 ; j=1,2)$

$$
v_{k j}^{0-}=0 .
$$




\section{§7. DÉTERmination EXPLICITE DU CHAMP DES DÉPLACEMENTS ET DES CONTRAINTES DES} DISLOCATIONS EXTRINSĖQUES

Il résulte de ce qui précède que le champ des déplacements des dislocations extrinsèques, dans le substrat, est strictement identique à celui des dislocations intrinsèques.

Par contre, dans la couche épitaxique, le champ des déplacements diffère de celui des dislocations intrinsèques par des termes additifs, linéaires en $x_{1}$ et $x_{2}$, suivant l'éqn. $(2 b)$. En résolvant le système formé par les éqns. $(53 b),(54 b)$ et $(54 c)$, nous obtenons les expressions

$$
v_{k 1}^{0+}=-\frac{b_{k}}{\Lambda}(k=1,2,3) ; \quad v_{12}^{0+}=\frac{b_{2}}{\Lambda} ; \quad v_{22}^{0+} \quad\left(1-\frac{v^{+} b_{1}}{-v^{+}}\right) \Lambda^{1} \quad v_{32}^{0+}=0 .
$$

En insérant ces relations dans l'éqn. $(54 a)$, les contraintes moyennes non nulles dans la couche sont les suivantes:

$$
\sigma_{11}^{0+}=\frac{-2 \mu^{+} b_{1}}{\left(1-v^{+}\right) \Lambda} \quad \sigma_{33}^{0+} \quad \frac{-2 \mu^{+} v^{+} b_{1}}{\left(1-v^{+}\right) \Lambda}, \quad \sigma_{13}^{0+} \quad \frac{-\mu^{+} b_{3}}{\Lambda}
$$

Ce résultat confirme l'éqns. (15) de Willis et aì. (1990).

§8. Calcul de l’énergie Élastique stockée $E$ pour une famille de dislocations INTRINSÈQUES PARALLÈLES, EN FONCTION DE L'ÉPAISSEUR $h$ DE LA COUCHE

Une coupure est tout d'abord effectuée le long de l'interface $x_{2}=0$. Pour rétablir les liaisons atomiques, il faut exercer des contraintes de surface. Le travail des forces de surface correspondantes est calculé pour le déplacement relatif indiquée par l'éqn. $(10 a)$. Dans cette intégrale, les contraintes $\sigma_{2 k}$ sont exprimées par l'éqns. (48)-(50) relatives au milieu, calculées pour $x_{2}=0$. Formellement, elles sont plus simples à écrire pour le milieu $x_{2}<0$ à cause des eqns. (9). La surface d'intégration s'étend sur une longueur unité le long de $\mathrm{O} x_{3}$, et le long de $\mathrm{O} x_{1}$ sur l'intervalle $\left(r_{0}, \Lambda-r_{0}\right), r_{0}$ étant le rayon de coupure (Hirth et Lothe 1982). L'énergie élastique stockée par unité de surface, notée $E_{\mathrm{s} 1}$, est donc

$$
E_{\mathrm{S} 1}=\frac{1}{2 \Delta} \int_{r_{0}}^{A-r_{0}} b_{k}\left(-\sigma_{2 k}^{-}\right)_{x_{2}}=0\left(\frac{x_{1}}{\Lambda}-\frac{1}{2}\right) \mathrm{d} x_{1} \text {. }
$$

L'intégration donne le résultat suivant:

$E_{\mathrm{S} 1}=\frac{\mu^{-} \omega k}{\pi} \sum_{1}^{\infty}\left(\frac{4 N_{1}}{\Delta}+\frac{(1-\exp (-2 h n \omega)) b_{3}^{2}}{1+k+(1-k) \exp (-2 h n \omega)}\right)\left[\left(1-\frac{2 r_{0}}{1}\right) \frac{\cos \left(n \omega r_{0}\right)}{4 n \pi}+\frac{\sin \left(n \omega r_{0}\right)}{4 n^{2} \pi^{2}}\right]$

avec $\Delta$ donnée par l'éqn. $(31 b)$ et

$$
\begin{aligned}
N_{1}= & \left.b_{1}^{2}+b_{2}^{2}\right)\left[1-v^{+}-k+k v^{-}+\left(-1+v^{+}-k+k v^{-}\right)\right. \\
& \times \exp (4 h n \omega)]+\left[\left(b_{1}^{2}+b_{2}^{2}\right) 2 k\left(1-v^{-}\right)\left(1+2 h^{2} n^{2} \omega^{2}\right)\right. \\
& \left.+\left(b_{2}^{2}-b_{1}^{2}\right) 4 h n \omega\left(1-v^{+}\right)\right] \exp (2 h n \omega) .
\end{aligned}
$$

L'exactitude des éqns. (56b) et (56c) a été vérifiée en intégrant numériquement (56a) avec des données correspondant au système $\mathrm{GaSb} / \mathrm{GaAs}(\S 10)$. 
§9. CHAMP ÉlastiQUE D'Un RÉSEAU CARRÉ DE DISLOCATIONS INTRINSÈQUES

Grâce au principe de superposition, le champ élastique de deux réseaux de dislocations intrinsèques sur la même interface peut être résolu simplement. A titre d'exemple, le champ élastique d'un réseau carré de dislocations est calculé ci-dessous.

Notons $u_{k}\left(x_{1}, x_{2}, x_{3}\right)$ le champ des déplacements lié au réseau de dislocations de la famille I (fig. 2) pour laquelle les dislocations ont un vecteur de Burgers $b_{k}$. Pour noter les champs règnant dans chacun des milieux, il suffit d'affecter un indice supérieur + ou - , selon le cas, aux symboles correspondant à l'un ou l'autre milieu.

Construisons une autre famille de dislocations de Vernier, la famille II, déduite de la précédente par rotation de $+\pi / 2$ autour de $\mathrm{O} x_{2}$. Au point $\mathrm{M}^{\prime}\left(-x_{3}, x_{2}, x_{1}\right)$ correspond donc le point $\mathbf{M}\left(x_{1}, x_{2}, x_{3}\right)$. Le champ des déplacements total se déduit de l'addition des champs des familles I et II. La contribution de la famille II est donnée par l'équation matricielle suivante, où $u_{k}^{\mathrm{l}}$ est donné par les équa. (36)-(41):

$$
\left[u_{k}^{\mathrm{II}}(\mathbf{M})\right]=[R]\left[\begin{array}{c}
u_{1}^{\mathrm{I}}\left(\mathbf{M}^{\prime}\right) \\
u_{2}^{\mathrm{I}}\left(\mathbf{M}^{\prime}\right) \\
u_{3}^{\mathrm{I}}\left(\mathbf{M}^{\prime}\right)
\end{array}\right]=\left[\begin{array}{c}
u_{3}^{\mathrm{I}}\left(\mathbf{M}^{\prime}\right) \\
u_{2}^{\mathrm{I}}\left(\mathbf{M}^{\prime}\right) \\
-u_{1}^{\mathrm{I}}\left(\mathbf{M}^{\prime}\right)
\end{array}\right]
$$

avec

$$
E_{\mathrm{S} 2}=\frac{1}{2 \Lambda^{2}} \int_{r_{0}}^{\Lambda-r_{0}} \int_{r_{0}}^{\Lambda-r_{0}}\left(-\sigma_{2 k}^{(1+\mathrm{II})}\right)_{x_{2}=0}\left[\left(u_{k}^{+}-u_{k}^{-}\right)^{\mathrm{I}}+\left(u_{k}^{+}-u_{k}^{-}\right)^{\mathrm{II}}\right] \mathrm{d} x_{1} \mathrm{~d} x_{3} .
$$

Le champ des déplacement total obtenu est donc

$$
\left[\begin{array}{l}
u_{1} \\
u_{2} \\
u_{3}
\end{array}\right]=\left[\begin{array}{l}
u_{1}^{\mathrm{I}}(\mathrm{M})+u_{3}^{\mathrm{I}}\left(\mathrm{M}^{\prime}\right) \\
u_{2}^{\mathrm{I}}(\mathrm{M})+u_{2}^{\mathrm{I}}\left(\mathrm{M}^{\prime}\right) \\
u_{3}^{\mathrm{l}}(\mathrm{M})-u_{1}^{\mathrm{I}}\left(\mathrm{M}^{\prime}\right)
\end{array}\right] \text {. }
$$

Fig. 2

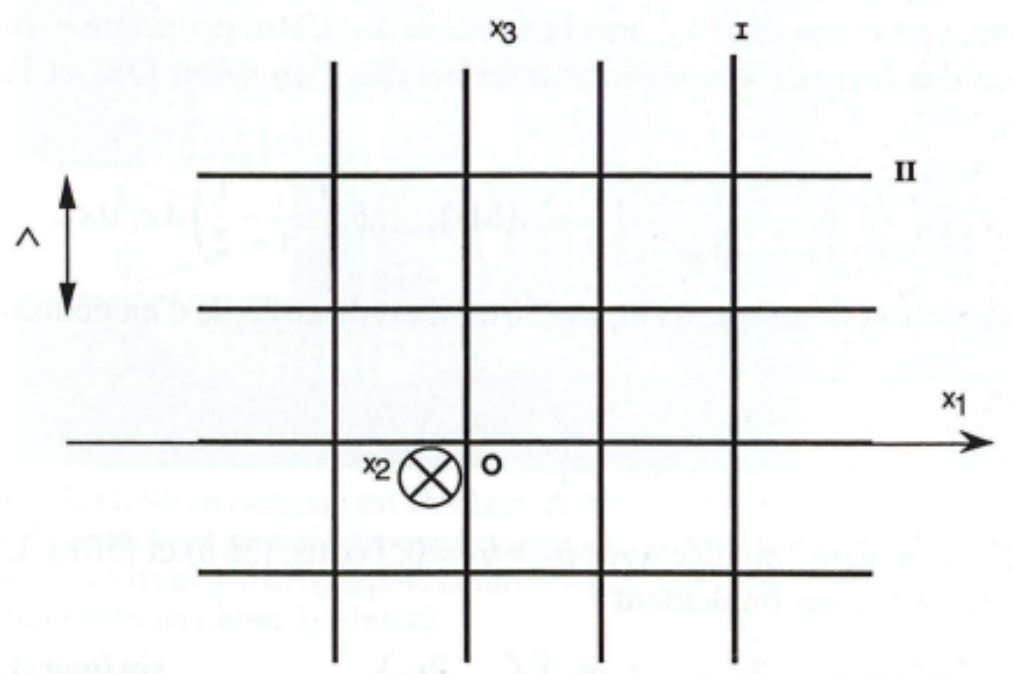

Schéma d'un réseau de dislocations à motif carré, formé de deux familles I et II. Les dislocations sont orientées respectivement selon les axes $\mathrm{O} x_{3}$ et $\mathrm{O} x_{1}$. La période est $\Lambda$. 
Pour le champ des contraintes, la contribution de la famille II est calculéc d'une façon analogue. En notant $\sigma_{i,}^{\prime}(\mathrm{M})(i, j=1,2,3)$ le champ des contraintes en $\mathrm{M}\left(x_{1}, x_{2}, x_{3}\right)$ de la famille 1 , l'éqns. (15), nous obtenons

$$
\left[\sigma_{i j}^{\prime \prime}(\mathrm{M})\right]=[R]\left[\sigma_{i j}^{l}\left(\mathrm{M}^{\prime}\right)\right][R]^{-1}=\left[\begin{array}{ccc}
\sigma_{33}^{1}\left(\mathrm{M}^{\prime}\right) & \sigma_{23}^{1}\left(\mathrm{M}^{\prime \prime}\right. & -\sigma_{13}^{1}\left(\mathrm{M}^{\prime}\right) \\
\sigma_{23}^{1}\left(\mathrm{M}^{\prime}\right) & \sigma_{22}^{1}\left(\mathrm{M}^{\prime}\right) & -\sigma_{12}^{1}\left(\mathrm{M}^{\prime}\right) \\
-\sigma_{13}^{1}\left(\mathrm{M}^{\prime}\right) & -\sigma_{12}^{\mathrm{I}}\left(\mathrm{M}^{\prime}\right) & \sigma_{11}^{1}\left(\mathrm{M}^{\prime}\right)
\end{array}\right] . \quad(58 a)
$$

Le champ des contraintes total obtenu est alors

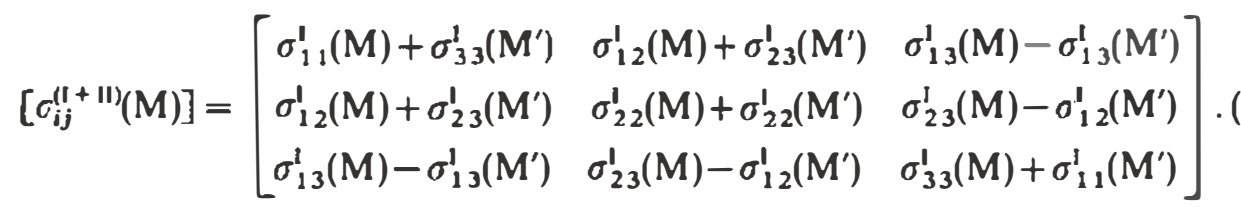

L’énergic élastique emmagasinéc par unité de surface, noléc $E_{S 2}$, est calculéc en intégrant le travail des forces de surfaces le long d'un carré de côté $\Lambda-2 r_{0}$ et en le rapportant à la surface $\Lambda^{2}$ (éqn. (57b)).

Ce calcul est eflectué ci-dessous dans le cas où les dislocations sont purement coins. Cette intégrale se simplifie alors beaucoup puisque les déplacements relatifs $\left(u_{k}^{+}-u_{k}^{-}\right)^{t}$ sont parallèles à l'axe $\mathrm{O} x_{1}$, les déplacements relatif́s $\left(u_{k}^{+}-u_{k}^{-}\right)^{\prime \prime}$ sont parallèles à l'axc $O x_{3}$, ct que, pour la famille I, $b_{2}=b_{3}=0$. L'intégrale s'écrit dans ce cas

$E_{\mathrm{S} 2}=\frac{1}{2 \Lambda^{2}} \int_{r_{0}}^{\Lambda-r_{0}} \int_{r_{0}}^{\Lambda-r_{0}}\left\{\left[-\sigma_{21}^{\mathrm{I}}(\mathrm{M})\right]_{x_{2}=0}\left(u_{1}^{+}-u_{1}^{-}\right)^{\mathrm{I}}+\left[\sigma_{23}^{\mathrm{II}}(\mathrm{M})\right]\left(u_{3}^{+}-u_{3}^{-}\right)^{\mathrm{II}}\right\} \mathrm{d} x_{1} \mathrm{~d} x_{3}$.

Compte tenu des éqns. (45), (57a) et ( $58 a)$, de la variation en dents de scie du déplacement relatif $(10 a)$, et de

$$
\left(u_{1}^{+}-u_{1}^{-}\right)^{\mathrm{II}}=-b_{1}\left(\frac{-x_{3}}{\Lambda}+\frac{1}{2}\right)=b_{1}\left(\frac{x_{3}}{\Lambda}-\frac{1}{2}\right),
$$

il s'ensuit que l'intégrale (59) se découple en deux intégrales de même valeur, avec, sous le signe somme, soit la variable $x_{1}$, soit la variable $x_{3}$. Cette propriété exprime en fait l'indépendance des travaux des cissions interfaciales, l'un selon $\mathrm{O} x_{1}$ et l'autre selon $\mathrm{O} x_{3}$. Au total

$$
E_{\mathrm{S} 2}=\frac{1}{\Lambda^{2}} \int_{r_{0}}^{\Lambda-r_{0}} \int_{r_{0}}^{\Lambda-r_{0}}\left[-\sigma_{21}^{\prime}(\mathrm{M})\right]_{x_{2}=0} b_{1}\left(\frac{x_{1}}{\Lambda}-\frac{1}{2}\right) \mathrm{d} x_{1} \mathrm{~d} x_{3}
$$

L'intégration en $x_{\mathrm{t}}$ est identique à l'équa. (56a). Il est donc facile d'en déduire le résultat suivant:

$$
E_{\mathrm{S} 2}=2\left(1-\frac{2 r_{0}}{1}\right) E_{\mathrm{S} 1}
$$

pour lequel $E_{s 1}$ doit être calculéc avec $b_{2}=b_{3}=0$, l'éqns. ( $\left.56 b\right)$ et $(56 c)$. L'expression complète de $E_{\mathrm{S} 2}$ est donc finalement

$$
E_{\mathrm{S} 2} \frac{2 \mu^{-} \omega k}{\pi^{2}}\left(1-\frac{2 r_{0}}{\Lambda}\right) b_{1}^{2} \sum_{1}^{\infty} \frac{N_{2}}{n \Delta}\left[\left(1-\frac{2 r_{0}}{\lambda}\right) \cos \left(n \omega r_{0}\right)+\frac{\sin \left(n \omega r_{0}\right)}{n \pi}\right],
$$


où $\Delta$ est donné par l'éqn. $(31 b)$ avec les expressions reportées à l'Annexe, et où

$$
\begin{aligned}
N_{2}= & 1-v^{+}-k+k v^{-}+\left(-1+v^{+}-k+k v^{-}\right) \exp (4 h n \omega) \\
& +\left[2 k\left(1-v^{-}\right)\left(1+2 h^{2} n^{2} \omega^{2}\right)-4 h n \omega\left(1-v^{+}\right)\right] \exp (2 h n \omega) .
\end{aligned}
$$

\section{§10. Application aux Interfaces épitaxiQues $\mathrm{GaSb} / \mathrm{GaAs}$ et $\mathrm{GaAs} / \mathrm{Si}$}

\subsection{GaSb/GaAs}

La croissance epitaxique de très fines couches de GaSb sur un substrat de GaAs dont la surface est orientée (001) a été étudiée par plusieurs équipes, dont Rocher et al. (1990a) et Bourret et al. (1991). Les réseaux cubiques de ces cristaux sont disposés parallèlement. La figure 3 est une micrographie électronique en faisceau faible obtenue par Rocher et al. (1990b). Elle met en évidence des défauts linéaires qui se répètent avec une périodicité de $5.6 \mathrm{~nm}$ le long de deux directions orthogonales $\langle 100\rangle$. Selon ces auteurs, l'origine de ces défauts n'est pas reliée à un mécanisme conventionnel de source de dislocations qui glisseraient suivant des plans (111), hypothèse qui conduirait à une nature extrinsèque de ces dislocations interfaciales. En fait, les expériences de diffraction des RX de Bourret et al. (1991) ont permis d'établir la nature intrinsèque de ces dislocations en comparant, indirectement, le champ des déplacements expérimentaux avec le champ théorique de dislocations intrinsèques (les équa. (32a) and (32 c) † de Bonnet (1981)). Le motif carré de ces défauts est observable dès qu'une épaisseur de $1 \mathrm{~nm}$ est obtenue. La nature intrinsèque des dislocations a été également confirmée par des simulations du champ des déplacements des colonnes atomiques, observable sur des images de microscopie électronique à haute résolution (Loubradou 1990).

Dans ce paragraphe, nous appliquons l'eqn. (63) pour examiner l'influence de l'épaisseur $h$ sur la valeur de l'énergie élastique stockée par unité de surface. Les

Fig. 3

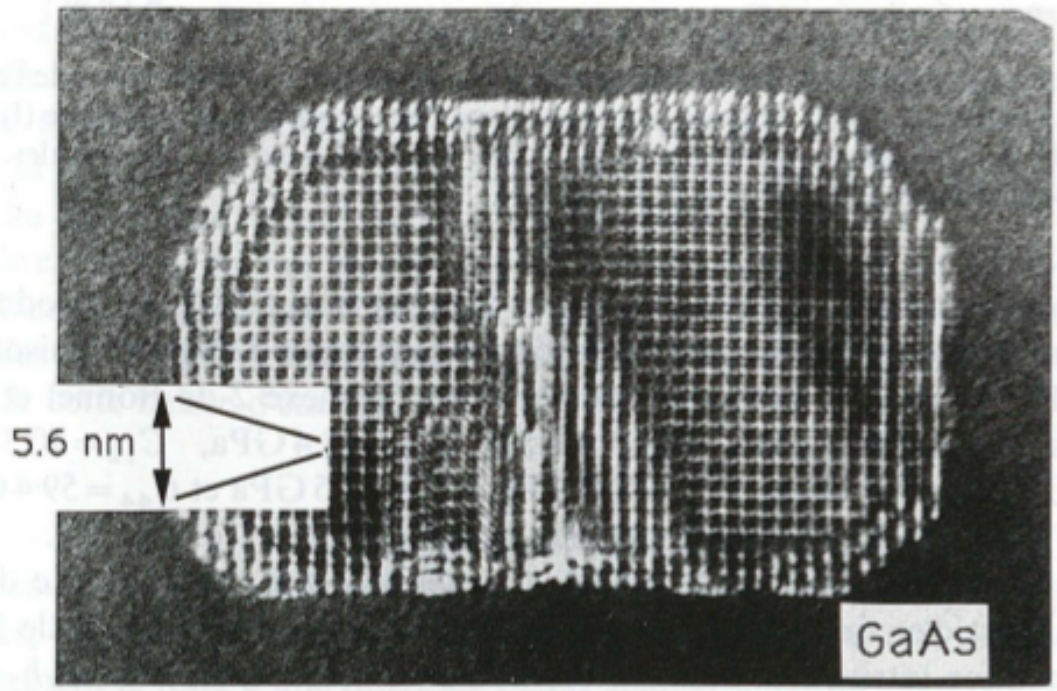

Aspect d'un îlot de GaSb en épitaxie sur une surface (001) de GaAs. L'interface est tapissée d'un réseau bipériodique de dislocations intrinsèques purement coin, formant un motif carré de côté $\Lambda=5.6 \mathrm{~nm}$. (Micrographie communiquée par A. Rocher, CEMES, Laboratoire d'Optique Electronique Toulouse).

† Dans l'éqn. (32c), le coefficient de l'exponentiel de $F_{6}$ doit être $+2 \pi g\left|x_{2}\right|$. 
Paramètres cristallins et modules d’élasticité utilisés dans les calculs de l'énergic élastique emmagasinćc (figs. 4 et 5).

\begin{tabular}{llcc}
\hline & $\begin{array}{c}a \\
(\mathrm{~nm})\end{array}$ & $\begin{array}{c}\mu \\
(\mathrm{GPa})\end{array}$ & $v$ \\
$\mathrm{GaSb}$ & 0.6096 & 33.9 & 026 \\
$\mathrm{GaAs}$ & 0.5653 & 46.0 & 0.25 \\
$\mathrm{Si}$ & 0.54282 & 66.1 & 0.23 \\
\hline
\end{tabular}

Fig. 4

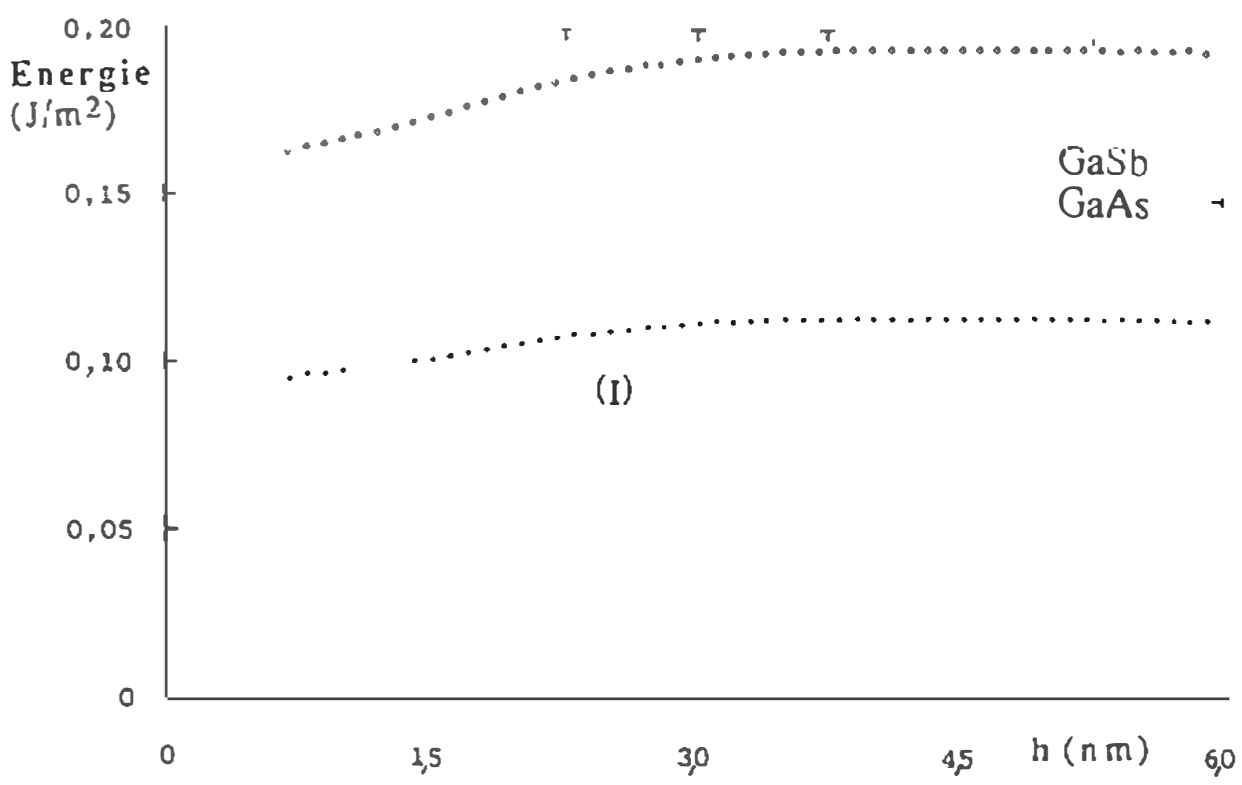

Varia:ion des énergies ćlastiques emmagasinécs, par unité de surlace, en fonction de l'ćpaisseur $h$ de la couche de GaSb déposéc sur GaAs dans deux hypothèses: la courbe (I), une scule famille de dislocations intrinsèques; la courbe(II), deux familles orthogonales. La courbe (II) se déduit de la courbe (I) par l'éqn. (62!

paramc̀tres des cristaux sont ceux donnés par Bourret et al. (1990). Les modules $\mu$ et $v$ (tableau) sont déterminés par le calcul à partir des constantes d'elasticité anisotropes de Hearmon (1979), selon la méthode indiquée dans l'Annexe 2 de Bonnet et Morton (1987). Ces constantes sont, pour GaSb $C_{11}=88.4 \mathrm{GPa}, C_{12}=40.3 \mathrm{GPa}$ et $C_{44}=43.4 \mathrm{GPa}$ et, pour GaAs, $C_{11}=118 \mathrm{GPa}, C_{12}=53.5 \mathrm{GPa}$ et $C_{44}=59.4 \mathrm{GPa}$. Les modules de cisaillement obtenus sont dans le rapport $k=\mu^{+} / \mu^{-}=0.737$.

Dans cet exemple, GaSb est le cristal + et GaAs est le cristal - . Le désaccord paramétrique est d'environ $7 \%$; la période est $\Lambda=5.6 \mathrm{~nm}$. Pour la famille I, (fig. 2), chaque dislocation intrinsèque a un vecteur de Burgers $\left(b_{1}=-0.3997 \mathrm{~nm} ; b_{2}=b_{3}=0\right)$; le rayon de coupure est $r_{0}=\left|b_{1}\right|$.

La figure 4 indique les variations avec l'épaisseur $h$ des énergies élastiques stockées, dans l'hypothèse d'une seule famille de dislocations (I), puis de deux familles orthognnales (11) comme ceci est visihle sur la fiz. 3. C.sénergies atteignent des valeurs asymptotiques respectivement égales à 114 et $195 \mathrm{~mJ} \mathrm{~m}^{-2}$, dès que l'épaisseur atteint $5 \mathrm{~nm}$ environ. 
Fig. 5

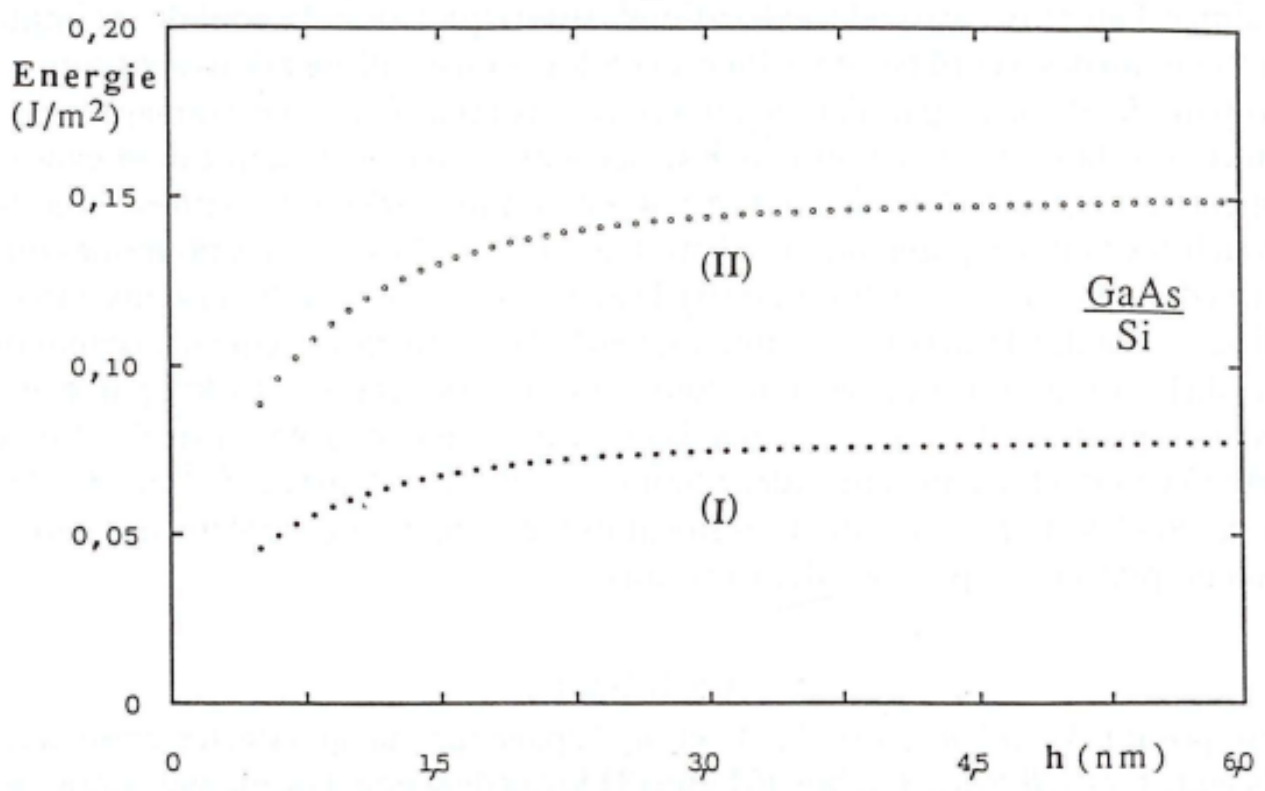

Même chose que pour la fig. 4 , mais pour le système $\mathrm{GaAs} / \mathrm{Si}$.

10.2. $\mathrm{GaAs} / \mathrm{Si}$

Ce système a déjà fait l'objet de plusieurs études lorsque le silicium est le substrat orienté (001), par exemple celles de Gerthsen, Ponce, Anderson et Chung (1988) et Rocher, Charasse, Bartenlian et Chazelas (1990a). Les dislocations intrinsèques de cette interface sont les mêmes que précédemment. Les constantes d'élasticité anisotropes sont, pour $\mathrm{Si}$ (Hirth et Lothe 1982), $C_{11}=165.7 \mathrm{GPa}, C_{12}=63.9 \mathrm{GPa}$ et $C_{44}$ $=79 \cdot 6 \mathrm{GPa}$.

Les modules d'élasticité isotropes sont calculés comme précédemment. Le rapport des modules de cisaillement est plus petit que précédemment puisque $k=0 \cdot 696$. Le paramètre cristallin du silicium (Cullity 1967) conduit ici à $b_{1}=-0.3838 \mathrm{~nm}$. La période, qui permet de rattraper le désaccord paramétrique de $4 \%$, est calculée égale à $9 \cdot 7 \mathrm{~nm}$. Elle est en bon accord avec les observations.

Pour le calcul de l'énergie stockée (fig. 5), le rayon de coupure est aussi $r_{0}=\left|b_{1}\right|$. Les courbes (I) et (II) indiquent que l'énergie est plus faible que dans le cas précédent, malgré des modules d'élasticité supérieurs. La valeur de la période $\Lambda$ du motif carré est donc à l'origine de cette baisse d'énergie.

\section{$\S 11$. CONCLUSION}

Ce travail donne la solution explicite complète du champ élastique (déplacements, contraintes, dilatations volumiques, énergie) d'une famille de dislocations réparties périodiquement le long d'une interface plane, celle-ci séparant un cristal (d'épaisseur finie $h$ ) en épitaxie sur un substrat monocristallin semi-infini. Certains résultats obtenus récemment par Willis et al. (1990) pour des dislocations à caractère extrinsèque sont confirmés, notamment la présence dans la couche de contraintes moyennes dont les valeurs sont inversement proportionnelles à la période $\Lambda$. Notre analyse indique que le champ des déplacements des dislocations extrinsèques ne diffère de celui des dislocations intrinsèques que par l'addition de termes linéaires. 
La solution obtenue est appliquée pour le champ des déplacements et des contraintes d'un réseau carré de dislocations intrinsèques, dont la période est imposée par la valeur du désaccord paramétrique. Dans le cas où ces dislocations sont coin, avec un vecteur de Burgers parallèle à l'interface, l'énergie élastique emmagasinée est cxprimíe sous la forme d'une série de Fourier semi-convergente dépendant exponentiellement du rapport $\boldsymbol{h} / \boldsymbol{\Lambda}$. Deux applications numériques ont été présentées, l'une pour une couche de $\mathrm{GaSb}$ déposée sur un substrat de $(001)$ de $\mathrm{GaAs}$, l'aut re pour une couche de GaAs déposée sur un substrat de (001) de Si. Ces systèmes ont des réseaux cubiques parallèles et ont des désaccords paramétriques de 7 et $4 \%$ respectivement, accommodés par des dislocations intrinsèques coin. Pour $\mathrm{GaSb} / \mathrm{GaAs}$, l'énergie stockée par le réseau de dislocations ccsse de croître dès que l'épaisseur $\boldsymbol{h}$ de la couche est de l'ordre de la périodc $(5.6 \mathrm{~nm})$, et atteint une valeur limite voisine de $195 \mathrm{~mJ} \mathrm{~m}^{-2}$. Pour GaAs/Si, l'énergie stockée par le réscau de dislocations est inférieure, malgré des modules d’élasticité plus élevés pour les deux cristaux.

\section{A N NEXE}

En posant $k=\mu^{+} / \mu^{-}, \omega=2 \pi / \Lambda$, et $h$, l'épaisseur de la couche épitaxiée, les coefficients $t_{j i}$ et $\alpha, \beta, \gamma$ des $X_{j}$ (éqns. (31 $a$ )et ( $\left.31 b\right)$ qui dépendent de manière naturelle de $n \geqslant 1$, sont donnés par.

$$
\begin{aligned}
& t_{11}=0 \text {, } \\
& I_{12}=2\left[2-(h n \omega)^{2}-2 k+k(h n \omega)^{2}+3 k v^{-}+2 k v^{-}(h n \omega)-6 v^{+}+3 k v^{+}-2 k v^{+}(h n \omega)\right. \\
& \left.-4 k v^{+} v^{-}+4\left(v^{+}\right)^{2}\right] \\
& t_{13}=2 k\left(2-3 v^{-}-3 v^{+}+4 v^{+} v^{-}\right) \text {, } \\
& t_{14}=0 \text {, } \\
& l_{15}=-5-6(h n \omega)-2(h n \omega)^{2}+5 k+6 k(h n \omega)+2 k(h n \omega)^{2}-6 k v^{-}-4 k v^{-}(h n \omega)+12 v^{+} \\
& +8 v^{+}(h n \omega)-6 k v^{+}-4 k v^{+}(h n \omega)+8 k v^{+} v^{-}-8\left(v^{+}\right)^{2}, \\
& \imath_{16}=-3-5 k+6 k v^{-}+4 v^{+}+6 k v^{+}-8 k v^{+} v^{-} \text {, } \\
& t_{21}=0 \text {, } \\
& I_{22}=-3+2(h n \omega)+3 k-2 k(h n \omega)-4 k v^{-}+4 v^{+} \text {, } \\
& \iota_{23}=-1-3 k+4 k v^{-} \text {, } \\
& t_{24}=0 \text {, } \\
& l_{25}=3+2(h n \omega)-3 k-2 k(h n \omega)+4 k v^{-}-4 v^{+} \text {, } \\
& 1_{26}=1+3 k-4 k v^{-} \text {, } \\
& l_{31}=2 k\left(v^{-}-v^{+}\right) \text {, } \\
& 1_{32}=-2\left[2-(h n \omega)^{2}+4 k(h n \omega)-3 k(h n \omega)^{2}+k v^{-}-6 k v^{-}(h n \omega)+4 k v^{-}(h n \omega)^{2}-6 v^{+}\right. \\
& \left.-k v^{+}-6 k v^{+}(h n \omega)+8 k v^{+} v^{-}(h n \omega)+4\left(v^{+}\right)^{2}\right] \text {, } \\
& t_{33}=0 \text {, } \\
& l_{34}=-3+3 k-2 k v^{-}+4 v^{+}-2 k v^{+} \text {, } \\
& \imath_{33}=-5+6(h n \omega)-2(h n \omega)^{2}-3 k+10 k(h n \omega)-6 k(h n \omega)^{2}+2 k v^{-}-12 k v^{-}(h n \omega) \\
& +8 k v^{-}(h n \omega)^{2}+12 v^{+}-8 v^{+}(h n \omega)+2 k v^{+}-12 k v^{+}(h n \omega)+16 k v^{+} v^{-}(h n \omega)-8\left(v^{+}\right)^{2} \text {, } \\
& \iota_{36}=0 \text {, } \\
& 1_{41}=-1+k \text {, } \\
& I_{42}=-3-2(h n \omega)-k-6 k(h n \omega)+8 k v^{-}(h n \omega)+4 v^{+} \text {, } \\
& l_{43}=0 \text {, } \\
& 1_{44}=-1+k \text {, } \\
& l_{43}=-3+2(h n \omega)-k+6 k(h n \omega)-8 k v^{-}(h n \omega)+4 v^{+} \text {. } \\
& 1_{46}=0 \text {, } \\
& i_{31}=2 k\left(v^{-}-v^{+}\right) \text {, } \\
& l_{32}=-4 k\left[1+2(h n \omega)-2(h n \omega)^{2}-v^{-}-4 v^{-}(h n \omega)+2 v^{-}(h n \omega)^{2}-2 v^{+}-2 v^{+}(h n \omega)\right. \\
& \left.+2 v^{+} v^{-}+4 v^{+} v^{-}(h n \omega)\right] \text {, } \\
& t_{33}=2 k\left(2-3 v^{-}-3 v^{+}+4 v^{+} v^{-}\right) \text {, }
\end{aligned}
$$




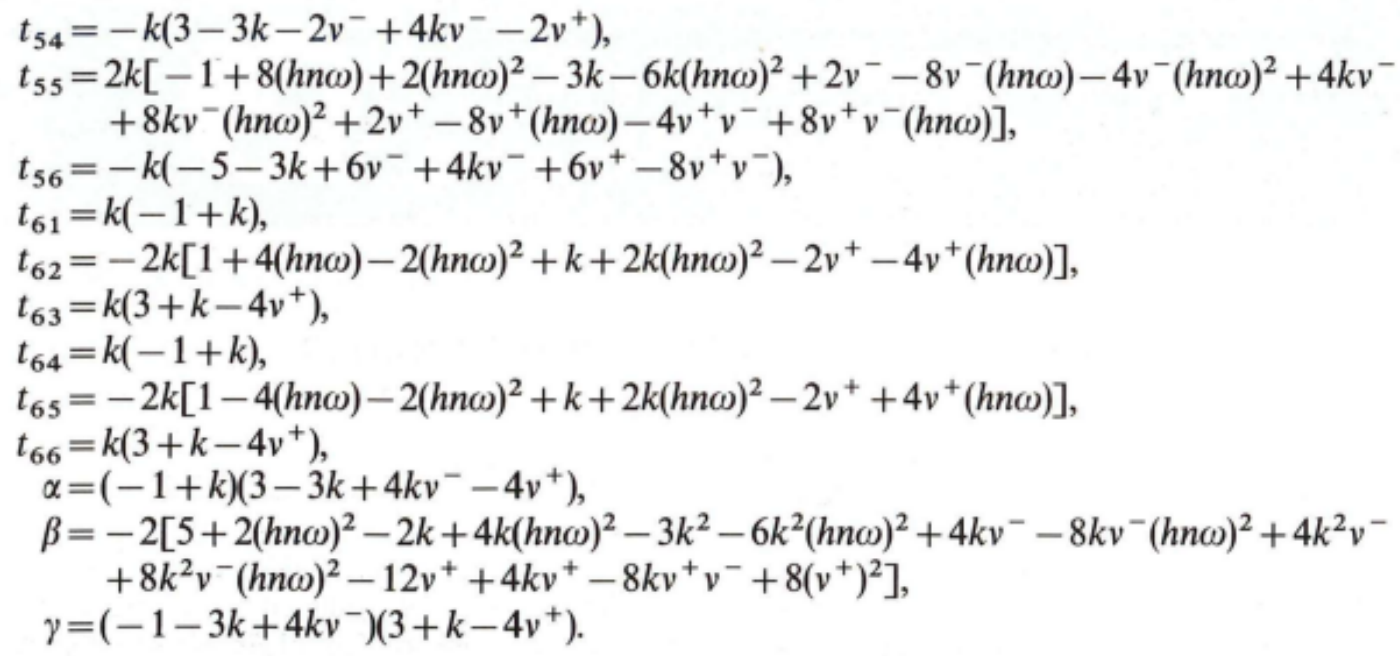

\section{RÉFÉRENCES}

Bass, J., 1964, Cours Mathématiques, 1, 297.

BONNET, R., 1981, Phil. Mag. A, 43, 1165.

Bonnet, R., Loubradou, M., Catana, A., et Stadelmann, P., 1991, Metall. Trans. A, 22, 1145.

Bonnet, R., et Morton, A. J., 1987, Phil. Mag. A, 56, 815.

Bourret, A., Fuoss, P. H., Rocher, A., et Raisin, C., 1991, Advances in Surface and Thin Film Diffraction, édité par T. C. Huang, P. I. Cohen, et D. J. Eaglesham, Vol. 208 (Pittsburgh, Pennsylvania: Materials Research Society), p. 303.

Bulle-Lieuwma, C. W. T., van Ommen, A. H., et Hornstra, J., 1988, High Resolution Electron Microscopy of Defects in Materials, édité par R. Sinclair, D. J. Smith, et U. Dahmen, Materials Research Society Symposium Proceedings, Vol. 102 (Pittsburgh, Pennsylvania: Materials Research Society), p. 377.

Catana, A., 1990, Thése 864, Ecole Polytechnique Fédérale de Lausanne.

Catana, A., Schmid, P. E., Heintze, M., Lévy, F., Stadelmann, P., et Bonnet, R., 1990, J. Appl. Phys., 67, 1820.

Cherns, D., Smith, D. A., Krakow, W., et-Batson, P. E., 1982, Phil. Mag. A, 45, 107.

Cullity, B. D., 1967, X-ray Diffraction, (Reading, Massachusetts: Addison-Wesley), p. 484.

FEuIllet, G., 1990, Evaluation of Advanced Semiconductor Materials by Electron Microscopy, édité par D. Cherns (New York: Plenum), p. 33.

Frank, F. C., et van der Merwe, J. H., 1949, Proc. R. Soc. A, 198, 205, 216.

Friedel, J., 1979, Dislocations in Solids, Vol. 1, édité par F. R. N. Nabarro (Amsterdam: NorthHolland), p. 1.

Gerthson, F. A., Ponce, F. A., Anderson, G. B., et Chung, H. F., 1988, Interfacial Structure, Properties, and Design, édité par M. H. Yoo, W. A. T. Clark, et C. L. Briant, Materials Research Society Symposium Proceedings, Vol. 122 (Pittsburgh, Pennsylvania: Materials Research Society), p. 21.

Gibson, J. M., Tung, R. T., Phillips, J. M., et Hull, R., 1985, J. Phys., Paris, 46, C4-369.

HiRTH, J. P., et Lothe, J., 1982, Theory of Dislocations, deuxième édition (New York: Wiley).

HeArmon, R. F. S., 1979, Numerical Data and Functional Relationship in Science and Technology, Landolt-Bernstein, New Series, Vol. 11, édité par K.-H. Hellwege and A. M. Hellwege (Berlin: Springer), p. 27.

Loubradou, M., 1990, Thèse, Institut National Polytechnique de Grenoble.

PÉnISSON, J. M., 1991, J. Phys. Materials III, 1, 1015.

Rocher, A., Charasse, M. N., Bartenlian, B., et Chazelas, J., 1990a, Proceedings of the International Congress on Intergranular and Interphase Boundaries in Materials, Paris, 48 September 1989 (Paris: Les Éditions de Physique, Av. du Hoggar, ZI de Courtaboeuf, BP 112, France t. 51, C1-915.

Rocher, A., Da Silva et C. Raisin, 1990b, Rev. Phys. Appl., 25, 957.

Tietz, L. A., Summerfelt, S. R., et CARTer, C. B., 1990, Atomic Scale Structure of Interfaces, édité par R. D. Bringans, R. M. Feenstra, et J. M. Gibson, Materials Research Society Symposium Proceedings, Vol. 159 (Pittsburgh, Pennsylvania, Materials Research Society), p. 209.

VAn der Merwe, J. H., 1982a, Phil. Mag. A, 45, 127; 1982b, Ibid., 45, 145; 1982c, Ibid., 45, 159.

Willis, J. R., Jain, S. C., et Bullough, R., 1990, Phil. Mag. A, 62, 115. 